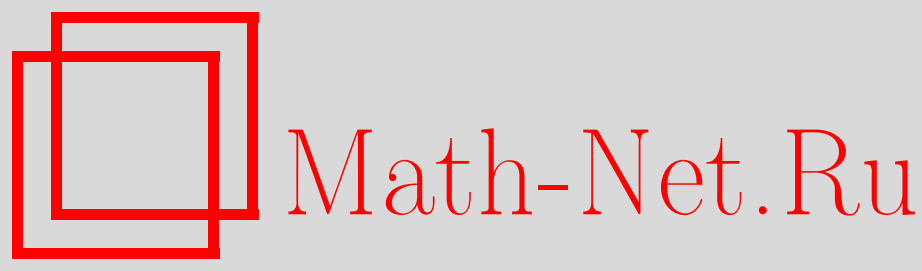

В. М. Бухштабер, С. И. Тертычный, Голоморфные решения дважды конфлюентного уравнения Гойна, ассоциированного с RSJ-моделью перехода Джозефсона, TMФ, 2015, том 182, номер 3, 373-404

DOI: https://doi.org/10.4213/tmf8770

Использование Общероссийского математического портала Math-Net.Ru подразумевает, что вы прочитали и согласны с пользовательским соглашением http://www . mathnet.ru/rus/agreement

Параметры загрузки:

IP : 54.237 .206 .68

26 апреля 2023 г., 12:21:35

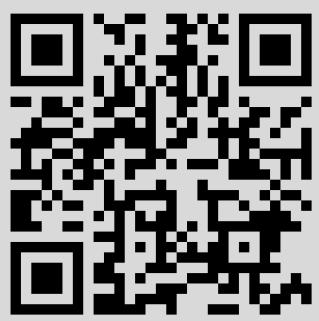




\title{
ФИЗИКА
}

Том 182, № 3

март, 2015

(C) 2015 г. $\quad$ В. М. Бухштабер*, С. И. Тертычный ${ }^{\dagger}$

\section{ГОЛОМОРФНЫЕ РЕШЕНИЯ ДВАЖДЫ КОНФЛЮЕНТНОГО УРАВНЕНИЯ ГОЙНА, АССОЦИИРОВАННОГО C RSJ-МОДЕЛЬЮ ПЕРЕХОДА ДЖОЗЕФСОНА}

\begin{abstract}
Работа представляет собой продолжение исследования нелинейного дифференциального уравнения первого порядка, используемого для моделирования сильношунтированного перехода Джозефсона. В основу подхода положена связь этого уравнения с дважды конфлюентным уравнением Гойна - линейным однородным дифференциальным уравнением второго порядка с двумя иррегулярными особыми точками. Описаны условия на параметры этого уравнения, при которых его общее решение представляет собой аналитическую функцию на сфере Римана без 0 и $\infty$. Построен в явном виде базис пространства решений, состоящий из пары функций, одна из которых голоморфна всюду, кроме бесконечности, а вторая - всюду, кроме нуля. Показано, что в рамках RSJ-модели динамики перехода Джозефсона описанная ситуация, когда возникает однозначность всех решений дважды конфлюентного уравнения Гойна на сфере Римана без 0 и $\infty$, соответствует условию обращения в нуль ширины ступеньки Шапиро.
\end{abstract}

Ключевые слова: дважды конфлюентное уравнение Гойна, голоморфные решения, динамическая система на торе с тождественным отображением Пуанкаре.

DOI: $10.4213 / \operatorname{tmf} 8770$

\section{1. ВВЕДЕНИЕ}

Настоящая работа содержит доказательства и обсуждение результатов, изложенных в заметке [1], которые опираются на связь между нелинейным дифференциальным уравнением первого порядка

$$
\dot{\varphi}(t)+\sin \varphi(t)=B+A \cos \omega t
$$

* Математический институт им. В. А. Стеклова РАН, Москва, Россия. E-mail: buchstab@mi.ras.ru

†ФГУП "Всероссийский научно-исследовательский институт физико-технических и радиотехнических измерений”, Менделеево, Московская обл., Россия 
где $A>0, B, \omega>0$ - вещественные константы, точкой обозначена производная по вещественной переменной $t$, и линейным однородным дифференциальным уравнением второго порядка

$$
z^{2} E^{\prime \prime}+\left((l+1) z+\mu\left(1-z^{2}\right)\right) E^{\prime}+(-\mu(l+1) z+\lambda) E=0
$$

где $E=E(z)$ - аналитическая в некоторой области функция, штрих обозначает производную по комплексной переменной $z$. Здесь константы $\lambda, \mu, l$ связаны с предыдущими соотношениями:

$$
l=\frac{B}{\omega}, \quad \mu=\frac{A}{2 \omega}, \quad \lambda=\left(\frac{1}{2 \omega}\right)^{2}-\mu^{2},
$$

и также вещественны. Уравнение (1) встречается в составе ряда моделей в физике, механике и геометрии [2], [3]. Наиболее широкую известность оно получило в составе так называемой резистивной модели перехода Джозефсона в сверхпроводниках [4]-[8], возникая в тех случаях, когда емкостью перехода можно пренебречь. С уравнением (1) связана известная динамическая система на торе, важная в теории быстро-медленных систем [9]. В свою очередь, уравнение (2) представляет собой подсемейство также достаточно хорошо известного семейства дважды конфлюентных уравнений Гойна [10], [11].

В работе показано, что каждое решение уравнения (1) порождает решение уравнения (2) - некоторую функцию, аналитическую на римановой поверхности функции логарифм либо на факторпространстве этой поверхности по некоторой подгруппе группы $\mathbb{Z}$, включая рассматриваемый здесь более подробно случай римановой сферы с удаленными полюсами. Решение уравнения (2) определено с точностью до умножения на вещественную константу.

Показано, что верно и обратное утверждение: любое ненулевое решение уравнения (2) порождает решение уравнения (1) с точностью до слагаемого, целократного $2 \pi$.

Мы анализируем ситуацию, когда параметр $l$ является целым числом. Показано, что в этом случае при выполнении некоторых условий, налагаемых на постоянные параметры, уравнение (2) допускает решения, голоморфные в окрестностях своих особых точек, $z=0$ или $z=\infty$. Отметим, что данный результат получен методом, дающим явную конструкцию решений с указанными свойствами.

При этом общее решение данного уравнения оказывается однозначной аналитической функцией на сфере Римана с удаленными полюсами. В терминах свойств соответствующих решений уравнения (1) это означает периодичность с периодом $T=2 \pi \omega^{-1}$ за вычетом приращения функции $\varphi(t)$ на величину, целократную $2 \pi$. Данное свойство решений уравнения (1) означает, что при выделенных значениях параметров для динамической системы на торе, ассоциированной с этим уравнением [12], [13], отображение Пуанкаре является тождественным. Выделим в евклидовом пространстве $\mathbb{R}^{3}$ с координатами $B, A, \omega$ подмножества, объединяющие точки с общим значением числа вращения. Зонами захвата фазы называются открытые множества в $\mathbb{R}^{3}$, замыкания которых совпадают с каким-либо из указанных подмножеств. В работе [13] показано, что для уравнения (1) зоны захвата фазы возникают только для целых чисел вращения. Сечения зон захвата плоскостями $\omega=$ const paзбиваются на непересекающиеся открытые множества, замыкания которых попарно 


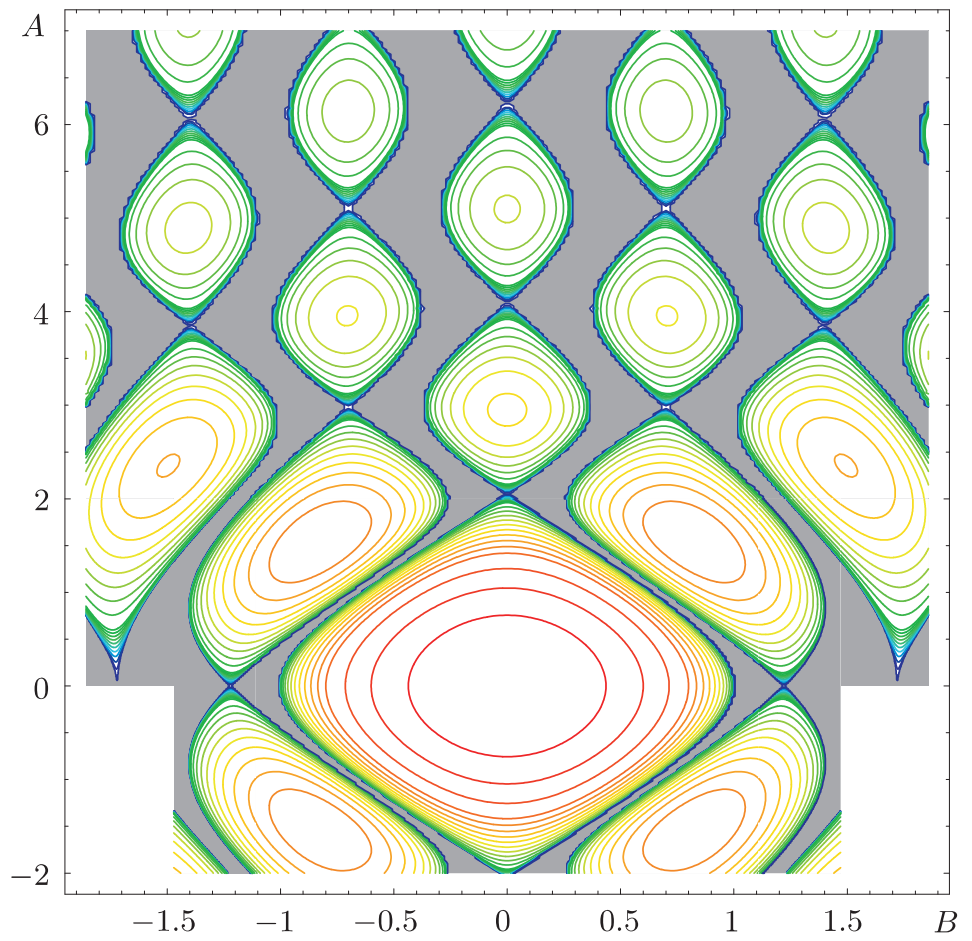

Рис. 1. Пример сечения областей захвата фазы плоскостью $\omega=$ const (в данном случае $\omega=0.7$ ).

пересекаются не более чем в одной точке. Есть основания полагать, что замыкание зоны захвата фазы, соответствующее одному общему числу вращения, образует фигуру, получаемую "перетяжками" из вертикальной полосы (см. рис. 1). Более того, можно показать, что ширина этой полосы убывает при удалении от горизонтали, и "перетяжки" идут с примерно постоянным шагом [14]. В терминах RSJ-модели1) перехода Джозефсона значения параметров, дающие "перетяжку", определяют ситуации, когда ширина ступеньки Шапиро равна нулю.

На рис. 1 изображены сечения областей захвата фазы плоскостью $\omega=$ const. Данные подмножества выделяются общим свойством определяемых ими решений уравнения (1). Точкам зоны захвата фазы соответствуют существование двух "по существу периодических" решений, одно из которых является устойчивым, а другое неустойчивым, и асимптотическая экспоненциальная сходимость всех остальных решений к устойчивому решению. Под по существу периодическими понимаются решения, представляющие собой сумму периодической и линейной функций. Число вращения $N$ определяется линейным вкладом, который имеет специальный вид, а именно равен $2 \pi \omega N t$, причем $N$ - целое число. Обратим внимание, что "перетяжка" на горизонтальной оси $B$, существующая при ненулевом числе вращения, отклонена по направлению от центра и является единственной, не находящейся на общей вертикальной прямой, на которой располагаются остальные "перетяжки".

\footnotetext{
1) Резистивно шунтированный переход Джозефсона.
} 
Уравнению (1) соответствует система из двух линейных дифференциальных уравнений с периодическими коэффициентами [15], [16]. На рис. 1 показаны линии постоянного уровня показателя Ляпунова данной системы в областях, где он существует (является вещественным), или, что то же самое, где наблюдается упомянутая выше асимптотическая сходимость к по существу периодическому решению для уравнения (1). При таком подходе легко идентифицируется граница областей захвата фазы. Она выделяется нулевым значением показателя Ляпунова. Область, где асимптотическая сходимость отсутствует (ее открытая часть), дополнительная к замыканию зон захвата, выделена однородным фоном. Для ее точек поведение всех решений уравнения (1) можно назвать псевдохаотическим. Это означает, что на множестве параметров полной меры решение не проявляет каких-либо признаков периодичности на любых интервалах изменения $t$, а на множестве параметров меры ноль периодичность определенного решения, наблюдаемая после вычитания некоторой линейной функции, не является устойчивой относительно сколь угодно малого возмущения параметров и начальных условий. Употребление термина "псевдохаотическое поведение" здесь уместно, поскольку демонстрируемое отсутствие какой-либо повторяемости на самом деле есть специфическое проявление развития некоторого периодического процесса, значения которого "считываются" функцией $\varphi(t)$ через промежутки "времени" $t$, несоизмеримые с его периодом. Для псевдохаотического поведения решений число вращения является нецелым. Имеет место формальное соответствие: целое число вращения - признак захвата фазы (кроме точек границ зон захвата), нецелое число вращения - признак псевдохаотического поведения.

Зоны захвата фазы на рис. 1 представляют собой цепочки деформированных вблизи горизонтальной координатной оси веретенообразных фигур, каждая соседняя пара которых соединена в одной точке, в окрестности которой их граница описывается парой гладких кривых, пересекающихся в этой точке. Каждая цепочка соответствует общему числу вращения, являющемуся целым. На вертикальную ось координат "нанизана" цепочка с числом вращения $N=0$. Для нее единственной пересечение с горизонтальной осью координат представляет собой не точку, а множество с непустой внутренностью, а именно отрезок $(-1,1)$. Правее расположены цепочки с числом вращения сначала $N=1$, а затем $N=2$; видны также фрагменты цепочки с $N=3$. Левее координатной оси расположены цепочки областей захвата фазы с числами вращения обратного знака, т.е. последовательно $N=-1,-2,-3$ (от последней, как и от зоны с $N=3$, в поле изображения попали только небольшие фрагменты).

Рассматриваемые фигуры на плоскости симметричны относительно начала координат (и отражения относительно обеих осей), поэтому, несмотря на то что часть рисунка в нижней координатной полуплоскости показана неполностью, ее легко восстановить.

Обратим внимание на расположение точек "перетяжек" на цепочках компонент зон захвата. Они могут быть выделены условием нулевой ширины пересечения зоны захвата с горизонтальной прямой $A=$ const. Как было показано недавно [17] (см. также [18], [19]), в представлении, соответствующем рис. 1, и в наших обозначениях все они, кроме попадающих на горизонтальную координатную ось, обладают значением абсциссы $B=l \omega$ для целого $l$, модуль которого не может превосходить модуля числа вращения. Численные расчеты свидетельствуют о том, что значения 
абсциссы "перетяжек" вне горизонтальной координатной оси совпадают с числом вращения.

Как оказывается, "перетяжки” соответствуют тем точкам в пространстве значений параметров, которые обеспечивают существование голоморфного решения уравнения (2).

В связи с этим отметим, что в работе сформулировано ограничение на параметры, являющееся достаточным при целом $l$ для существования решения уравнения (2), аналитического в окрестности точки $z=0$. На самом деле такое решение, которое мы будем для краткости называть голоморфным, представляет собой целую функцию. На его основе явным образом строится и решение, аналитическое в окрестности точки $z=\infty$ (и тогда аналитическое всюду, кроме точки $z=0$ ). В итоге для уравнения строится в явном виде общее решение - функция, аналитическая на сфере Римана вне точек $z=0$ и $z=\infty$.

Голоморфные решения представлены в виде ряда, коэффициенты которого заданы явными формулами. Предложенное представление оказывается пригодным не только для формального анализа, но и для численных расчетов, по крайней мере демонстрационного характера.

Важной особенностью уравнения (2) является то, что вместо рассмотрения случая отрицательных $l$ удобно наряду с ним ввести уравнение

$$
z^{2} \widehat{E}^{\prime \prime}+\left((-l+1) z+\mu\left(1-z^{2}\right)\right) \widehat{E}^{\prime}+(\mu(l-1) z+\lambda) \widehat{E}=0,
$$

полученное из (2) заменой $l$ на $-l$. Рассматривая пару уравнений $(2)$ и $(4)$, мы можем полагать (и будем так поступать в дальнейшем), что $l \geqslant 0$. Оказывается, что голоморфные решения уравнения (4) существуют для тех же значений параметров $\lambda, \mu$, для которых они существуют и для уравнения (2). Обратное соответствие также имеется, но проявляет себя не столь прямолинейно. В связи с этим необходимо отметить, что при целых неотрицательных $l$ для уравнения (4) при некоторых значениях параметров $\lambda$ и $\mu$ могут существовать полиномиальные решения, т. е. функция $\widehat{E}(z)$ будет иметь вид полинома от переменной z [20]. (Уравнение (2), напротив, не может иметь полиномиальных решений.) Как показано в работе [21], уравнение (4) с параметрами $l, \lambda, \mu$ имеет полиномиальные решения тогда и только тогда, когда $(\lambda, \mu)$ является точкой явно описанной алгебраической кривой степени $l$ по $\lambda$ и $2[\mu / 2]$ по $\mu$.

В буквальном смысле полином является голоморфной функцией, однако семейство полиномиальных решений уравнения (4), по-видимому, в отличие от ситуации, типичной для классических ортогональных полиномов, не пересекается с рассматриваемым здесь "голоморфным" семейством, состоящим из функций, разлагаемых в бесконечный степенной ряд с неотрицательными показателями (ряд Тейлора). При целом $l$ из существования голоморфного решения уравнения (4) вытекает, что его общее решение представляет собой однозначную аналитическую функцию на сфере Римана вне точек $z=0$ и $z=\infty$; при существовании же полиномиального решения это не так.

Мы выдвигаем следующие гипотезы.

ГиПотезА 1. Если уравнение (4) обладает полиномиальным решением, то сопутствующее уравнение (2) не имеет решения, аналитического в нуле. 
ГиПотезА 2. Достаточное условие существования в нуле аналитического решения уравнения (2) (см. ниже теорему 2) является также и необходимым.

Справедливость гипотезы 1 влечет за собой справедливость гипотезы 2 (см. приложение Б).

Гипотеза 1 оказывается верной, если не обращаются в нуль определенные детерминанты порядка $l-1$, по одному для каждого $l$ (см. (53)), элементы матриц которых представляют собой модифицированные функции Бесселя первого рода от положительного аргумента [1]. Тот факт, что эти детерминанты не обращаются в нуль, очевиден при $l=1$, доказан при $l=2$ и подтвержден численно при нескольких последующих $l$.

Таким образом, для целых значений $l>2$ вопрос о существовании голоморфных решений уравнений (2), (4), не принадлежащих к семейству решений, описанному в настоящей работе, остается, строго говоря, открытым.

\section{2. ГОЛОМОРФНЫЕ РЕШЕНИЯ И ИХ СВОЙСТВА}

Очевидно, не требует отдельного доказательства тот факт, что любое решение уравнения (1) может быть продолжено на неограниченный интервал $-\infty<t<\infty$. Из этого факта, в частности, вытекает следующий результат.

Теорема 1. Любое решение $\varphi(t)$ уравнения (1) порождает аналитическую функuию - решение $E(z)$ уравнения (2), удовлетворяющую условию

$$
\overline{E(z)}=E(\bar{z})
$$

и определенную на римановой поверхности, представляющей собой универсальное накрытие плоскости комплексного переменного z без нуля (область определения функции логарифм), либо на его фактор-многообразии по группе $\mathbb{Z}$ поворотов на угол, кратный $2 \pi$. Функция $E(z)$ определена с точностью до умножения на ненулевое вещественное число. Наоборот, любое решение уравнения (2), удовлетворяющее условию (5), порождает некоторое решение уравнения (1), определенное с точностью до слагаемого, кратного $2 \pi$.

ДокАзАТЕЛьство. Пусть задано некоторое решение уравнения (1). Определим функции

$$
\begin{aligned}
& x(t)=\exp \left(\int \cos \varphi(t) d t\right) \cos \frac{1}{2} \varphi(t), \\
& y(t)=-\exp \left(\int \cos \varphi(t) d t\right) \sin \frac{1}{2} \varphi(t) .
\end{aligned}
$$

Легко убедиться, что при выполнении (1) они удовлетворяют следующей системе линейных однородных уравнений с периодическими коэффициентами [15], [16]:

$$
\begin{aligned}
2 \dot{x}(t) & =x(t)+(B+A \cos \omega t) y(t), \\
-2 \dot{y}(t) & =y(t)+(B+A \cos \omega t) x(t) .
\end{aligned}
$$


Забудем теперь на время о связи функций $x(t), y(t)$ с функцией $\varphi(t)$. Пусть они суть некоторое нетривиальное (т. е. не тождественно нулевое) решение системы (7). В соответствии с известными свойствами линейных однородных систем такое решение продолжаемо на весь неограниченный интервал $-\infty<t<\infty$. Кроме того, функции $x(t), y(t)$ не обращаются одновременно в нуль при любом $t$, поскольку в противном случае, т. е. если бы имели место равенства $x\left(t_{0}\right)=0, y\left(t_{0}\right)=0$ при некотором $t_{0}$, мы имели бы для системы (7) задачу Коши с нулевыми начальными данными, для которой единственное решение - тривиальное, когда $x(t) \equiv 0, y(t) \equiv 0$, т. е. случай, который мы исключаем из рассмотрения. Из данного замечания следует, что выражение

$$
\Phi(t):=\frac{x(t)-i y(t)}{x(t)+i y(t)}
$$

является гладкой функцией переменной $t$, причем $|\Phi(t)| \equiv 1$. Соответственно, мы можем определить вещественную функцию $\varphi(t)$ уравнением

$$
e^{i \varphi(t)}=\Phi(t)
$$

Тем самым функция $\varphi(t)$ определена однозначно с точностью до прибавления константы, целократной $2 \pi$.

Имея функцию $\varphi(t)$, определенную, как описано выше, на всей числовой оси, с помощью дифференцирования уравнения (9) можно показать, что она удовлетворяет уравнению (1), если функции $x(t), y(t)$ удовлетворяют системе $(7)$.

Таким образом, мы показали, что решения уравнения (1) и системы (7) находятся в "почти однозначном" взаимном соответствии. Неполная однозначность здесь означает возможность прибавления к $\varphi(t)$ константы, целократной $4 \pi$, без изменения функций $x(t), y(t)$; при прибавлении же нечетнократного $2 \pi$ числа функции $x(t), y(t)$ совместно меняют знак с сохранением выполнения определяющих их дифференциальных уравнений. Очевидно, что и более общее преобразование, умножение обеих функций $x(t), y(t)$ на ненулевое число, также на выполнении уравнений $(7)$ не сказывается и функцию $\varphi(t)$ не меняет.

Рассмотрим теперь систему линейных однородных уравнений

$$
\begin{aligned}
4 i \omega z^{2} X^{\prime}(z) & =2 z X(z)+\left(2 B z+A\left(z^{2}+1\right)\right) Y(z), \\
-4 i \omega z^{2} Y^{\prime}(z) & =\left(2 B z+A\left(z^{2}+1\right)\right) X(z)+2 z Y(z),
\end{aligned}
$$

где $z$ - комплексная переменная. Пусть областью изменения для $z$ будет некоторое открытое связное подмножество плоскости комплексного переменного, содержащее по крайней мере часть дуги окружности единичного радиуса $S^{1}=S_{1}^{1}$ (символом $S_{r}^{1}$ мы будем обозначать окружность радиуса $r>0)$, где функции $X(z), Y(z)$ являются аналитическими. Единственной особой точкой для уравнений (10) является центр $z=0$, поэтому в любой иной точке любое их решение аналитично и может быть продолжено через границу области изменения $z$, если таковая имеется и не сводится к точке $z=0$. Ниже мы увидим, какова должна быть эта область изменения $z$, чтобы такие продолжения уже не могли быть реализованы; пока же наше рассмотрение локальное. 
Выделим вещественные и мнимые части функций $X(z), Y(z)$ и преобразуем их к виду функций от двух полярных координат (интерпретируя $\operatorname{Re} z$ и $\operatorname{Im} z$ как декартовы координаты), введя следующие обозначения:

$$
\begin{gathered}
X(z)=: X_{\mathrm{R}}(r, \phi)+i X_{\mathrm{I}}(r, \phi), \\
Y(z)=: Y_{\mathrm{R}}(r, \phi)+i Y_{\mathrm{I}}(r, \phi), \\
z=: r e^{i \phi}, \quad X_{\mathrm{R}}, X_{\mathrm{I}}, Y_{\mathrm{R}}, Y_{\mathrm{I}}, \phi \in \mathbb{R}, \quad r \in \mathbb{R}^{+} .
\end{gathered}
$$

При сделанных предположениях в координатной полуплоскости $(r>0, \phi)$ существует прямоугольник, включающий некоторый отрезок "горизонтальной" прямой $r=1$ (возможно, всю эту прямую целиком), в котором функции $X_{\mathrm{R}}, X_{\mathrm{I}}, Y_{\mathrm{R}}, Y_{\mathrm{I}}$ определены и являются гладкими.

Воспользуемся независимостью значения производной аналитической функции от направления дифференцирования. Если выбрать направление постоянного $r$, то система (10) сводится к следующей системе:

$$
\begin{aligned}
2 \omega \frac{\partial}{\partial \phi} X\left(r e^{i \phi}\right) & =X\left(r e^{i \phi}\right)+\left(B+\frac{1}{2} A\left(r e^{i \phi}+r^{-1} e^{i \phi}\right)\right) Y\left(r e^{i \phi}\right), \\
-2 \omega \frac{\partial}{\partial \phi} Y\left(r e^{i \phi}\right) & =\left(B+\frac{1}{2} A\left(r e^{i \phi}+r^{-1} e^{i \phi}\right)\right) X\left(r e^{i \phi}\right)+Y\left(r e^{i \phi}\right) .
\end{aligned}
$$

В частности, на дуге $S_{1}^{1}$ для функций $x(t)=X\left(e^{i \omega t}\right), y(t)=Y\left(e^{i \omega t}\right)$ данная система превращается в систему (7). Следовательно, она допускает вещественные решения на всей вещественной оси $-\infty<\phi<\infty$, и мы можем записать следующие соотношения:

$$
\begin{aligned}
& X_{\mathrm{R}}(1, \phi)=x\left(\omega^{-1} \phi\right), \quad X_{\mathrm{I}}(1, \phi)=0, \\
& Y_{\mathrm{R}}(1, \phi)=y\left(\omega^{-1} \phi\right), \quad Y_{\mathrm{I}}(1, \phi)=0 .
\end{aligned}
$$

Теперь рассмотрим реализацию производных в уравнениях (10) как частных производных по радиальному направлению. В результате, разделяя вещественные и мнимые части в соответствии с (11), получаем систему уравнений

$$
\begin{aligned}
2 \omega r \partial_{1} X_{\mathrm{R}} & =X_{\mathrm{I}}+\left(B+\frac{1}{2} A\left(r+r^{-1}\right) \cos \phi\right) Y_{\mathrm{I}}+\frac{1}{2} A\left(r-r^{-1}\right) \sin \phi Y_{\mathrm{R}}, \\
2 \omega r \partial_{1} X_{\mathrm{I}} & =-X_{\mathrm{R}}-\left(B+\frac{1}{2} A\left(r+r^{-1}\right) \cos \phi\right) Y_{\mathrm{R}}+\frac{1}{2} A\left(r-r^{-1}\right) \sin \phi Y_{\mathrm{I}}, \\
2 \omega r \partial_{1} Y_{\mathrm{R}} & =-Y_{\mathrm{I}}-\left(B+\frac{1}{2} A\left(r+r^{-1}\right) \cos \phi\right) X_{\mathrm{I}}-\frac{1}{2} A\left(r-r^{-1}\right) \sin \phi X_{\mathrm{R}}, \\
2 \omega r \partial_{1} Y_{\mathrm{I}} & =Y_{\mathrm{R}}+\left(B+\frac{1}{2} A\left(r+r^{-1}\right) \cos \phi\right) X_{\mathrm{R}}-\frac{1}{2} A\left(r-r^{-1}\right) \sin \phi X_{\mathrm{I}},
\end{aligned}
$$

где $\partial_{i}, i=1,2, \ldots$, обозначает оператор частного дифференцирования функции нескольких аргументов по $i$-му аргументу; например,

$$
\partial_{1} X_{\mathrm{R}}:=\frac{\partial X_{\mathrm{R}}(r, \phi)}{\partial r}, \quad \partial_{1} X_{\mathrm{R}}\left(r_{1}, \phi_{1}\right):=\left.\frac{\partial X_{\mathrm{R}}(r, \phi)}{\partial r}\right|_{r=r_{1}, \phi=\phi_{1}} .
$$

Как и система (12), это фактически обыкновенные дифференциальные уравнения. Они линейны и однородны, причем все коэффициенты - непрерывные и гладкие 
функции при $r>0$. Рассматривая (13) как начальные данные для них при $r=1$, мы можем утверждать, что при каждом фиксированном $\phi$ система (14) обладает единственным решением, удовлетворяющим условиям (13), и такое решение определено на всем луче $r>0$. Более того, функции $X_{\mathrm{R}}(r, \phi), X_{\mathrm{I}}(r, \phi), Y_{\mathrm{R}}(r, \phi), Y_{\mathrm{I}}(r, \phi)$ являются гладкими не только по отношению к $r$, но и по отношению к $\phi$ (теорема о гладкой зависимости решения от параметров и начальных данных). Заметим также, что для решения задачи Коши (14), (13) очевидным образом справедливы следующие равенства:

$$
\partial_{1} X_{\mathrm{R}}(1, \phi)=\partial_{1} Y_{\mathrm{R}}(1, \phi)=\partial_{2} X_{\mathrm{I}}(1, \phi)=\partial_{2} Y_{\mathrm{I}}(1, \phi)=0 .
$$

Рассмотрим теперь линейные комбинации частных производных:

$$
\begin{array}{ll}
C_{X \mathrm{R}}=C_{X \mathrm{R}}(r, \phi):=\partial_{2} X_{\mathrm{R}}+r \partial_{1} X_{\mathrm{I}}, & C_{X \mathrm{I}}=C_{X \mathrm{I}}(r, \phi):=-\partial_{2} X_{\mathrm{I}}+r \partial_{1} X_{\mathrm{R}}, \\
C_{Y \mathrm{R}}=C_{Y \mathrm{R}}(r, \phi):=\partial_{2} Y_{\mathrm{R}}+r \partial_{1} Y_{\mathrm{I}}, & C_{Y \mathrm{I}}=C_{Y \mathrm{I}}(r, \phi):=-\partial_{2} Y_{\mathrm{I}}+r \partial_{1} Y_{\mathrm{R}} .
\end{array}
$$

Непосредственная проверка показывает, что если функции $X_{\mathrm{R}}, X_{\mathrm{I}}, Y_{\mathrm{R}}, Y_{\mathrm{I}}$ удовлетворяют уравнениям (14), то для функций (16) выполняются уравнения

$$
\begin{aligned}
2 \omega r \partial_{1} C_{X \mathrm{R}} & =-C_{X \mathrm{I}}-\left(B+\frac{1}{2} A\left(r+r^{-1}\right) \cos \phi\right) C_{Y \mathrm{I}}+\frac{1}{2} A\left(r-r^{-1}\right) \sin \phi C_{Y \mathrm{R}}, \\
2 \omega r \partial_{1} C_{X \mathrm{I}} & =C_{X \mathrm{R}}+\left(B+\frac{1}{2} A\left(r+r^{-1}\right) \cos \phi\right) C_{Y \mathrm{R}}+\frac{1}{2} A\left(r-r^{-1}\right) \sin \phi C_{Y \mathrm{I}}, \\
2 \omega r \partial_{1} C_{Y \mathrm{R}} & =C_{X \mathrm{I}}+\left(B+\frac{1}{2} A\left(r+r^{-1}\right) \cos \phi\right) C_{X \mathrm{I}}-\frac{1}{2} A\left(r-r^{-1}\right) \sin \phi C_{X \mathrm{R}}, \\
2 \omega r \partial_{1} C_{Y \mathrm{I}} & =-C_{X \mathrm{R}}-\left(B+\frac{1}{2} A\left(r+r^{-1}\right) \cos \phi\right) C_{X \mathrm{R}}-\frac{1}{2} A\left(r-r^{-1}\right) \sin \phi C_{X \mathrm{I}} .
\end{aligned}
$$

Кроме того, согласно (15) и (16) имеем

$$
C_{X \mathrm{R}}(1, \phi)=C_{X \mathrm{I}}(1, \phi)=C_{Y \mathrm{R}}(1, \phi)=C_{Y \mathrm{I}}(1, \phi)=0 .
$$

Из (17) и (18) (задача Коши для линейных однородных уравнений с нулевыми начальными данными) следует, что

$$
C_{X \mathrm{R}}(r, \phi)=C_{X \mathrm{I}}(r, \phi)=C_{Y \mathrm{R}}(r, \phi)=C_{Y \mathrm{I}}(r, \phi) \equiv 0
$$

при всех $r>0$. С другой стороны, обращение в нуль функций (16) эквивалентно выполнению уравнений Коши-Римана, записанных в полярных координатах $r, \phi$, для функций $X=X_{\mathrm{R}}+i X_{\mathrm{I}}, Y=Y_{\mathrm{R}}+i Y_{\mathrm{I}}$ аргументов $r, \phi$. Таким образом, решение задачи Коши (14), (13) определяет аналитические функции $X(z), Y(z)$, удовлетворяющие уравнениям (10), на римановой поверхности с локальной координатой $z=r e^{i \phi}$, которая является образом координатной полуплоскости $\{r>0,-\infty<\phi<\infty\}$. Данной римановой поверхностью является универсальное накрытие плоскости комплексного переменного с удаленным центром, представляющее собой также область определения функции логарифм. Это и есть естественная область определения решений уравнений (10) в общем случае. В специальных случаях решение может оказаться периодичным по $\phi$ с периодом, целократным $2 \pi$. Тогда областью определения функций $X(z), Y(z)$ становится факторпространство этой поверхности по 
подгруппе группы $\mathbb{Z}$ или по самой этой группе, т. е. в последнем случае это просто плоскость комплексного переменного без нуля.

Рассмотрим теперь всегда обратимое линейное преобразование функций, входящих в уравнение (10), следующего вида [19]:

$$
\begin{aligned}
& u(z)=(2 \omega z)^{-1} z^{-l / 2} e^{\mu\left(-z+z^{-1}\right) / 2}(X(z)+i Y(z)), \\
& v(z)=i z^{-l / 2} e^{\mu\left(-z+z^{-1}\right) / 2}(X(z)-i Y(z)) .
\end{aligned}
$$

Продифференцировав эти формулы, можно показать, что при выполнении уравнений (10)

$$
\begin{aligned}
& u^{\prime}=z^{-2}\left(-\left((l+1) z+\mu\left(z^{2}+1\right)\right) u-(2 \omega)^{-2} v\right) \\
& v^{\prime}=u
\end{aligned}
$$

Таким образом, как следствие (10), выполняется уравнение

$$
z^{2} v^{\prime \prime}+\left((l+1) z+\mu\left(z^{2}+1\right)\right) v^{\prime}+(2 \omega)^{-2} v=0,
$$

представляющее собой частный случай дважды конфлюентного уравнения Гойна [20], [11]. Наконец, после подстановки

$$
v(z)=e^{-\mu z} E(z)
$$

получаем уравнение $(2)^{2)}$.

Таким образом, любое решение уравнения (1) можно “поднять" до аналитической функции $E(z)$, удовлетворяющей уравнению (2) и определенной на универсальном накрытии плоскости комплексного переменного с выколотым центром. Наоборот, любая такая функция, удовлетворяющая условию $\overline{E(z)}=E(\bar{z})$, определяет некоторое вещественное решение уравнения (1).

Чтобы в этом убедиться, выразим функции $X(z), Y(z)$ через решение уравнения (2). В результате получим

$$
\begin{aligned}
& X(z)=\frac{1}{2} z^{l / 2} e^{-\mu\left(z+z^{-1}\right) / 2}\left(2 \omega z\left(E^{\prime}(z)-\mu E(z)\right)-i E(z)\right), \\
& Y(z)=-\frac{1}{2} i z^{l / 2} e^{-\mu\left(z+z^{-1}\right) / 2}\left(2 \omega z\left(E^{\prime}(z)-\mu E(z)\right)+i E(z)\right) .
\end{aligned}
$$

При построении решения уравнения (1) используются их значения на единичной окружности. А именно, согласно (13), (11) определяются функции $x(t), y(t)$, а затем по формулам (8), $(9)$ - функция $\varphi(t)$. При этом, для того чтобы функция $\varphi(t)$ была вещественна, требуется, чтобы на единичной окружности функции $X(z), Y(z)$ принимали вещественные значения. Для выражений (25) это свойство далеко не очевидно; более того, оно в общем случае и не имеет места. Тем не менее добиться нужного результата можно, и это делается следующим образом.

Задача состоит в построении решения уравнения (2), которое порождало бы по формулам вида (25) функции, вещественные на $S^{1}$. При этом мы предполагаем, что

${ }^{2)}$ Впервые на возникновение в контексте данной задачи дважды конфлюентного уравнения Гойна было указано в работе [22]. 
некоторое решение $E(z)$ данного уравнения уже известно. Основной используемый инструмент - преобразование, которое мы обозначим символом $\downarrow$, отображающее пространство решений уравнения (2) в себя. Справедлив следующий результат, который устанавливается непосредственным вычислением.

Лемма 1. Если Е(z) есть решение уравнения (2), то функиия

$$
\natural E(z):=(\downarrow E)(z):=2 \omega z^{-l-1}\left(E^{\prime}\left(z^{-1}\right)-\mu E\left(z^{-1}\right)\right)
$$

также удовлетворяет данному уравнению.

Таким образом, если $E(z)$ - решение уравнения (2), то любая линейная комбинация функций $E(z)$ и $\downarrow E(z)$ с постоянными коэффициентами также является решением этого уравнения. Возьмем в качестве такой линейной комбинации выражение

$$
\frac{1}{2}(1+i)\left(e^{i \alpha / 2} E(z)+e^{-i \alpha / 2} \downarrow E(z)\right),
$$

где $\alpha$ - произвольная вещественная константа. Подставив это выражение вместо $E(z)$ в уравнения (25) и исключив вторые производные с помощью уравнения (2), получим

$$
\begin{aligned}
X(z)= & e^{-\mu\left(z+z^{-1}\right) / 2}\left(\frac{1}{2}(1+i) e^{i \alpha / 2} z^{l / 2}\left(2 \omega z\left(E^{\prime}(z)-\mu E(z)\right)-i E(z)\right)+\right. \\
& \left.+\frac{1}{2}(1-i) e^{-i \alpha / 2} z^{-l / 2}\left(2 \omega z^{-1}\left(E^{\prime}\left(z^{-1}\right)-\mu E\left(z^{-1}\right)\right)+i E\left(z^{-1}\right)\right)\right), \\
Y(z)= & e^{-\mu\left(z+z^{-1}\right) / 2}\left(\frac{1}{2}(1-i) e^{i \alpha / 2} z^{l / 2}\left(2 \omega z\left(E^{\prime}(z)-\mu E(z)\right)+i E(z)\right)+\right. \\
& \left.+\frac{1}{2}(1+i) e^{-i \alpha / 2} z^{-l / 2}\left(2 \omega z^{-1}\left(E^{\prime}\left(z^{-1}\right)-\mu E\left(z^{-1}\right)\right)-i E\left(z^{-1}\right)\right)\right) .
\end{aligned}
$$

При условии выполнения равенства $E(z) \equiv \overline{E(\bar{z})}$ эти функции, очевидно, вещественны на единичной окружности, на которой $z^{-1}=\bar{z}$. (Если для функции $E(z)$ данное равенство не выполняется, вместо нее следует использовать одну из функций $(E(z)+\overline{E(\bar{z})}) / 2$ или $i(-E(z)+\overline{E(\bar{z})}) / 2$, которые также являются решениями уравнения (2), поскольку все его постоянные коэффициенты вещественны.) Ими и формулами (8), (9) описывается семейство вещественных решений уравнения (1), зависящих от вещественного параметра $\alpha$, причем для функции $\Phi(z)$ имеет место следующее представление:

$$
\Phi(z)=-z^{l} \frac{e^{i \alpha / 2} E(z)+2 i e^{-i \alpha / 2} \omega z^{-l-1}\left[E^{\prime}\left(z^{-1}\right)-\mu E\left(z^{-1}\right)\right]}{e^{-i \alpha / 2} E\left(z^{-1}\right)-2 i e^{i \alpha / 2} \omega z^{l+1}\left[E^{\prime}(z)-\mu E(z)\right]} .
$$

Теорема доказана.

\section{3. ПОСТРОЕНИЕ РЯДА ТЕЙЛОРА, УДОВЛЕТВОРЯЮЩЕГО УРАВНЕНИЮ}

Рассмотрим теперь, при каких условиях для целого $l \geqslant 0$ уравнение $(2)$ допускает решение, представляющее собой целую функцию аргумента $z$. Пусть [23]

$$
R_{m}:=\prod_{j=m}^{\infty} M_{j}, \quad M_{j}:=\left(\begin{array}{cc}
1+\frac{\lambda}{j(j-l)} & \frac{\mu^{2}}{j(j-l)} \\
1 & 0
\end{array}\right), \quad m=l+1, l+2, \ldots,
$$


где в бесконечных произведениях множители с бо́льшими значениями индекса располагаются правее сомножителей с меньшими индексами. Существование матриц $R_{m}$, т. е. сходимость бесконечных матричных произведений (30) при любых $\lambda$ и $\mu$, доказывается в лемме 6 , помещенной в приложение А. Из этой леммы также следует, что все элементы матриц $R_{m}$ при $m \geqslant l+1$ ограничены в совокупности (следствие 2 ).

Определим функцию

$$
\xi_{l}(\lambda, \mu)=\left(\begin{array}{ll}
\lambda & \mu^{2}
\end{array}\right) \cdot R_{l+1} \cdot\left(\begin{array}{l}
1 \\
0
\end{array}\right)
$$

Теорема 2. Для данных $\lambda$ и $е$ условие $\xi_{l}(\lambda, \mu)=0$ является достаточным для существования нетривиального (т.е. не равного тождественно нулю) голоморфного решения уравнения $E(z)(2)$. При этом $E(0) \neq 0^{3)}$.

ДокАЗАтЕЛЬСтво. Рассмотрим ряд

$$
E(z)=\sum_{k=0}^{\infty} a_{k} z^{k}, \quad z \in \mathbb{C}
$$

где не зависящие от $z$ вещественные коэффициенты $a_{k}$ определяются формулами

$$
a_{0}=\left(\begin{array}{ll}
1 & 0
\end{array}\right) \cdot R_{l+1} \cdot\left(\begin{array}{l}
1 \\
0
\end{array}\right), \quad a_{k}=\frac{\mu^{k}}{k !}\left(\begin{array}{ll}
0 & 1
\end{array}\right) \cdot R_{k+l} \cdot\left(\begin{array}{l}
1 \\
0
\end{array}\right), \quad k=1,2, \ldots .
$$

Легко убедиться, что формальный ряд вида (32) удовлетворяет уравнению (4) тогда и только тогда, когда выполнены уравнения

$$
\begin{gathered}
\lambda a_{0}+\mu a_{1}=0, \\
-(k+l) \mu a_{k-1}+(\lambda+k(k+l)) a_{k}+(k+1) \mu a_{k+1}=0, \quad k=1,2, \ldots .
\end{gathered}
$$

Если ввести дифференциальный оператор $\mathfrak{L}$ второго порядка, задающий левую часть уравнения (4), то при выполнении уравнений (34) при $k=1,2, \ldots$ имеет место равенство

$$
\mathfrak{L} E(z)=\xi_{l} .
$$

Покажем, что из определений (33) вытекают уравнения (34) при $k=1,2, \ldots$. Рассмотрим матричное выражение

$$
-k(k+l) M_{k-1+l} \cdot M_{k+l}+(\lambda+k(k+l)) M_{k+l}+\mu^{2} I,
$$

где $I$ - единичная матрица, которое при $\mu \neq 0$ тождественно равно

$$
\frac{k !}{\mu^{k}}\left[-\mu(k+l) \frac{\mu^{k-1}}{(k-1) !} M_{k-1+l} \cdot M_{k+l}+(\lambda+k(k+l)) \frac{\mu^{k}}{k !} M_{k+l}+\mu(k+1) \frac{\mu^{k+1}}{(k+1) !} I\right] .
$$

После его упрощения получится матрица с нулями в нижней строке. Это легко проверить прямым вычислением. Соответственно, его умножение слева на вектор-строку (l $\left.\begin{array}{l}0 \\ 1\end{array}\right)$ дает вектор-строку ( $\left.\begin{array}{ll}0 & 0\end{array}\right)$; это справедливо также и для выражения в квадратных скобках. Дополнительное умножение справа теперь уже на любой

\footnotetext{
3) При $\lambda \neq 0$, очевидно, также справедливо $E^{\prime}(0) \neq 0$.
} 
вектор-столбец дает просто ноль тождественно. Выберем в качестве такого правого множителя $R_{k+1+l} \cdot\left(\begin{array}{l}1 \\ 0\end{array}\right)$. Тогда, принимая во внимание тождества

$$
M_{k+l} \cdot R_{k+1+l}=R_{k+l}, \quad M_{k-1+l} \cdot M_{k+l} \cdot R_{k+1+l}=R_{k-1+l}
$$

и используя определения (33), получаем второе из уравнений (34).

Таким образом, нами установлено, что при выполнении условия $\xi_{l}(\lambda, \mu)=0$, налагаемого на параметры $\lambda, \mu$, формулы (32), (33) определяют решение уравнения (2) в виде ряда Тейлора, по крайней мере формального. Область его сходимости можно охарактеризовать, применив оценку сверху для значений его коэффициентов. Действительно, согласно следствию 2 леммы 6 (см. приложение А) элементы всех матриц $R .$. , входящие в формулы (33), ограничены в совокупности. Тогда из самих этих формул следует, что ряд (32) обладает мажорантой, в качестве которой выступает ряд Тейлора функции $e^{|\mu| z}$. Поэтому он сходится при любом $z$, действительно определяя голоморфное решение уравнения (2).

Кроме того, само значение предела $\lim _{m \rightarrow \infty} R_{m}$ и формула (33) говорят о том, что коэффициенты $a_{k}$ при достаточно больших $k$ не равны нулю (хотя и стремятся к нулю вследствие наличия множителя $\left.(k !)^{-1}\right)$. Следовательно, $E(z) \not \equiv 0$.

Последнее из заключений теоремы следует из того, что в предположении, что $E(0)=a_{0}=0$, первое из уравнений (34) позволяет получить $a_{1}=0$, а тогда остальные уравнения системы (34) последовательно определяют $a_{k}=0$ для всех остальных $k>1$, так что $E(z) \equiv 0$. Мы пришли к противоречию, означающему, что $E(0) \neq 0$. Тем самым теорема доказана полностью.

Теорема 3. Пусть уравнение (2), в котором l есть иелое неотрицательное число, обладает голоморбным решением $E(z)$. Тогда любое решение этого уравнения можно представить в виде $a E(z)+b \downarrow E(z)$, где $a, b$ - произвольные константы.

ДокАЗАТЕЛЬство. В силу леммы 1 и двумерности пространства решений уравнения (2) доказательства требует лишь свойство линейной независимости функций $E(z)$ и $ઘ E(z)$. Предположим, что справедливо обратное. В силу определений оно означало бы, что функции $z^{l+1} E(z)$ и $E^{\prime}\left(z^{-1}\right)-\mu E\left(z^{-1}\right)$ линейно зависимы (отличаются постоянным множителем). Но это заведомо не так, поскольку первая из них голоморфна в окрестности точки $z=0$, а вторая в этой точке сингулярна. Последнее следует из теоремы Лиувилля, а также из того, что $E^{\prime}\left(z^{-1}\right)-\mu E\left(z^{-1}\right) \not \equiv 0$, поскольку функция $E(z)=$ const $\cdot e^{\mu z}$ не является решением уравнения (2) при выполнении следующего из (3) соотношения $4 \omega^{2}\left(\lambda+\mu^{2}\right)=1$. Теорема доказана.

СлЕДСтвиЕ 1. Если l есть целое неотрицательное число, то выполнение уравнения $\xi_{l}(\lambda, \mu)=0$ для данных $l, \lambda, \mu$ является достаточным условием для того, чтобы все решения уравнения (2) являлись однозначными аналитическими функииями на сфере Римана с удаленными полюсами.

Обратимся теперь к уравнению (4). Как отмечалось выше, оно отличается от уравнения (2), помимо замены обозначения неизвестной функции $E(z)$ на $\widehat{E}=\widehat{E}(z)$, только противоположным знаком у $l$. Этот модифицированный вариант уравнения (2) вводится для удобства, чтобы использовать его для сопоставления решений, соответствующих одинаковым значениям параметров $\lambda, \mu$, но различным (а именно, отличающимся противоположными знаками) значениям параметра $l$. Использование такого двойника ("антипода" [1]) уравнения (2) означает, что мы можем 
полагать значения $l$ неотрицательными, поскольку, чтобы учесть отрицательные $l$, достаточно рассмотреть вместо (2) (с отрицательным параметром $l$ ) уравнение (4), где параметр $l$ положителен; но нужно помнить, что при $l=0$ они, естественно, совпадают, что сопряжено с некоторой спецификой данного случая. В дальнейшем обозначение $\widehat{E}(z)$ будет соответствовать, как правило, решению уравнения (4).

Для решений обоих уравнений (2) и (4) присуще следующее свойство единственности.

ТЕОРема 4. Если у уравнений (2) или (4) существует решение, аналитическое в точке $z=0$, то оно определено однозначно с точностъю до умножения на константу.

ДоказАтельство. Рассмотрим выражения

$$
W_{ \pm}(z):=z^{1 \pm l} e^{-\mu\left(z+z^{-1}\right)}\left(\mathcal{E}_{1}^{\prime}(z) \mathcal{E}_{2}(z)-\mathcal{E}_{1}(z) \mathcal{E}_{2}^{\prime}(z)\right)
$$

где под символом $\mathcal{E}$ подразумевается либо $E$ и выбирается верхний знак, либо $\widehat{E}$ и выбирается нижний знак. Соответственно в первом случае мы имеем дело с уравнением (2), а во втором - с уравнением (4). Проверяется непосредственно, что в том случае, когда обе функции $\mathcal{E}_{1}(z), \mathcal{E}_{2}(z)$ удовлетворяют соответствующему уравнению (2) либо (4), производная от функции $W_{ \pm}(z)$ обращается в нуль, и, таким образом, правая часть формулы (37) (“перенормированный" вронскиан) представляет собой на самом деле константу. Это константа не равна нулю в том и только в том случае, когда функции $\mathcal{E}_{1}(z)$ и $\mathcal{E}_{2}(z)$ линейно независимы. Но тогда они обе не могут быть аналитичными в точке $z=0$, поскольку иначе мы имели бы равенство

$$
W_{ \pm}^{-1} z e^{-\mu z}\left(\mathcal{E}_{1}^{\prime}(z) \mathcal{E}_{2}(z)-\mathcal{E}_{1}(z) \mathcal{E}_{2}^{\prime}(z)\right)=z^{\mp l} e^{\mu z^{-1}}
$$

в котором выражение слева в этой точке аналитично, а выражение справа - нет. Полученное противоречие доказывает теорему.

\section{4. ЛИНЕЙНЫЕ ОТОБРАЖЕНИЯ ПРОСТРАНСТВ РЕШЕНИЙ И ИХ ПРИМЕНЕНИЯ}

Введем аналогично оператору (26) линейные операторы b, :

$$
\begin{aligned}
& b f(z):=2 \omega z^{-l+1} e^{\mu\left(z+z^{-1}\right)}\left[f^{\prime}(-z)-\mu f(-z)\right], \\
& \diamond f(z):=e^{\mu\left(z+z^{-1}\right)} f\left(-z^{-1}\right) .
\end{aligned}
$$

На пространстве функций, аналитических на сфере Римана с удаленными полюсами, при целом $l$ они представляют собой линейные отображения этого пространства в себя.

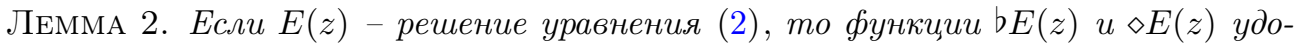
влетворяют уравнению (4).

Данный факт проверяется прямым вычислением.

Утверждение леммы 2 означает, что операторы $b, \diamond$ могут также интерпретироваться как гомоморфизмы из пространства решений уравнения (2) в пространство решений уравнения (4). 
Существуют и вполне аналогичные представимые в явном виде гомоморфизмы, действующие “в противоположном направлении". Для их описания введем еще один линейный оператор, обозначаемый символом d (“зеркальное отражение” символа b), на пространстве аналитических функций на сфере Римана с удаленными полюсами:

$$
\mathrm{d} f(z):=2 \omega z^{l+1} e^{\mu\left(z+z^{-1}\right)}\left[f^{\prime}(-z)-\mu f(-z)\right] .
$$

Формально выражение в правой части (40) отличается от выражения в правой части (38) только знаком перед параметром $l$, но как оператор на пространстве решений рассматриваемых уравнений оно имеет иной смысл.

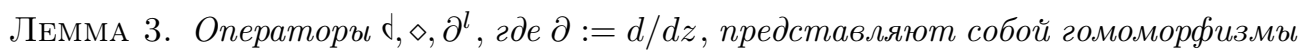
из пространства решений уравнения (4) в пространство решений уравнения (2).

ДокАзАтЕльство. Что касается операторов $d, \diamond$, это их свойство устанавливается прямой проверкой, как и аналогичное свойство операторов $b, \diamond$ в лемме 2 выше (более того, для оператора $d$ соответствующее формальное вычисление получается из вычисления, связанного с $b$, с помощью замены $l$ на $-l$ ). Для оператора же $\partial^{l}$ оно следует из тождеств

$$
\mathfrak{L}_{l} \equiv \mathfrak{L} \circ \partial^{l} \equiv \partial^{l} \circ \hat{\mathfrak{L}},
$$

связывающих при $k=l$ дифференциальные операторы

$$
\begin{aligned}
\mathfrak{L}_{k}:= & z^{2} \partial^{k+2}+\left((2 k-l+1) z+\mu\left(1-z^{2}\right)\right) \partial^{k+1}+ \\
& +(k(k-l)+\lambda-\mu(2 k-l+1) z) \partial^{k}-\mu k(k-l) \partial^{k-1}, \quad k=1,2,3 \ldots, \\
\mathfrak{L}:= & z^{2} \partial^{2}+\left((l+1) z+\mu\left(1-z^{2}\right)\right) \partial+(\lambda-\mu(l+1) z), \\
\hat{\mathfrak{L}}:= & z^{2} \partial^{2}+\left((-l+1) z+\mu\left(1-z^{2}\right)\right) \partial+(\lambda+\mu(l-1) z) .
\end{aligned}
$$

Первое из них есть следствие определения $\mathfrak{L}_{k}$, а второе может быть доказано, например, с помощью математической индукции. Лемма доказана.

Заметим, что, пренебрегая формальной строгостью, здесь также можно было бы считать, не внося каких-либо противоречий, что $\hat{\mathfrak{L}}=\mathfrak{L}_{0}$, если условиться полагать равным нулю последнее слагаемое в формуле для $\mathfrak{L}_{k}$, записываемое в данном случае как произведение $\mu \cdot 0 \cdot(-l) \cdot \partial^{-1}$, в котором смысл "интегрирования" $\partial^{-1}$ остается неопределенным.

ЛЕмма 4. Если уравнение (2) обладает решением, голоморфным на плоскости комплексного переменного, то все решения уравнения (4) представляют собой аналитические функции на плоскости комплексного переменного с удаленным нулем.

ДокАЗАтЕльство. Если уравнение (2) обладает голоморфным решением $E(z)$, то функции $b E(z), \diamond E(z)$ являются аналитическими всюду, кроме точки $z=0$, причем обе они не равны тождественно нулю. Эти функции линейно независимы. Действительно, согласно определениям (38), (39) из линейной зависимости $b E(z)$ и $\diamond E(z)$ следовала бы линейная зависимость функций $E\left(z^{-1}\right)$ и $z^{l+1}\left(E^{\prime}(z)-\mu E(z)\right)$. Но это невозможно, поскольку при голоморфном решении $E(z)$ первая функция сингулярна в точке $z=0$, а вторая аналитична (и не является тождественным нулем). Таким образом, поскольку размерность пространства решений уравнения (4) равна 2, пара его линейно независимых решений $b E(z), \diamond E(z)$ образует базис и любое решение 
представляет собой линейную комбинацию этих двух решений. Каждое из них есть аналитическая функция на плоскости комплексного переменного без нуля; соответственно, таким же будет и общее решение уравнения (4). Лемма доказана.

ТЕОрема 5. Если уравнение (2) обладает решением, аналитическим в точке $z=0$, то уравнение (4) также допускает решение, аналитическое в точке $z=0$.

В соответствии с теоремой 4 и то, и другое решение определены однозначно с точностью до умножения на константу.

ДоКАЗАТЕЛЬСТВо теОРЕмЫ 5. Рассмотрим подпространство двумерного линейного пространства решений уравнения (2), являющееся образом также двумерного линейного пространства решений уравнения (4) под действием отображения $\partial^{l}$. Ядро данного отображения нетривиально тогда и только тогда, когда уравнение (4) обладает решением в виде полинома порядка, не превосходящего $l-1$. Такой полином, если он существует, и будет решением, аналитическим в точке $z=0$.

Если же на решениях уравнения (4) ядро оператора $\partial^{l}$ тривиально, то задаваемое им отображение является эпиморфизмом. Тогда его образ содержит, в частности, голоморфную функцию $E(z)$, существующую в соответствии с условиями теоремы. Но поскольку $\partial^{l}$ есть кратное дифференцирование, то соответствующий прообраз решение уравнения (4), о котором заранее известно, что это функция, аналитическая всюду, кроме, возможно, нуля, - на самом деле голоморфно везде, включая ноль. Теорема доказана.

Теорема 6. Если уравнение (4) обладает решением, аналитическим в точке $z=0$ и не являющимся полиномом от $z$, то уравнение (2) также допускает решение, аналитическое в точке $z=0$ (голоморфное решение).

Действительно, таким решением будет функция $\partial^{l} \widehat{E}(z)$. Она не является тождественным нулем, поскольку $\widehat{E}(z)$ не является полиномом и удовлетворяет уравнению (2) в соответствии с леммой 3. (Заметим, что если бы решение $\widehat{E}(z)$ было полиномом, то мы могли бы получить таким способом для уравнения (2) только решение, тождественно равное нулю, что, конечно, никоим образом не устанавливает существование решения, аналитического в нуле.)

Оговорка насчет "неполиномиальности" в теореме 6 является существенной. Дело в том, что при целом положительном $l=n+1, n=0,1,2, \ldots$, уравнение (4), в отличие от уравнения (2), действительно может допускать решение, представляющее собой полином ${ }^{4)}$ от $z$ степени $n$ [19], [20]. Здесь также возникает ограничение на значения параметров $\lambda, \mu$, но оно имеет вид алгебраического уравнения, в отличие от требования обращения в нуль трансцендентной функции $\xi_{l}(\lambda, \mu)$, возникающего при рассмотрении голоморфных решений.

А именно, если определить матрицы $H_{n}$ с помощью формул

$$
\left(H_{n}\right)_{j k}=(n-j) \mu \delta_{j, k-1}-j(n+1-j) \delta_{j, k}+j \mu \delta_{j, k+1}, \quad j, k=0,1, \ldots, n,
$$

\footnotetext{
4) Хотя это и не доказано, есть основания утверждать, что полиномиальным решениям на диаграмме, изображенной на рис. 1 , соответствуют точки пересечения границ областей захвата фазы с вертикальными прямыми $B=\omega l$, единственные, не являющиеся точками "перетяжек", которые все также лежат на этих прямых; они расположены симметрично на границах связных подмножеств зон захвата, непосредственно примыкающих к горизонтальной оси координат (кроме границы открытого множества, включающего начало координат).
} 
где $\delta_{j, k}$ - символ Кронекера, то полиномиальные решения уравнения (4) существуют тогда и только тогда, когда

$$
\operatorname{det}\left(H_{n}+\lambda I\right)=0
$$

где $I$ - единичная матрица.

Например, при $n=0,1,2$ решениями уравнения (4) являются функции $E=P_{n}$, где

$$
\begin{aligned}
& P_{0}=1 \\
& P_{1}=\mu z-\lambda+1 \\
& P_{2}=-2 \mu^{2} z^{2}+2 \mu(\lambda-2) z-\lambda^{2}+4 \lambda+2 \mu^{2}-4
\end{aligned}
$$

и параметры $\mu, \lambda$ должны удовлетворять уравнениям $\Delta_{n}=0$ для

$$
\begin{aligned}
& \Delta_{0}=\lambda, \\
& \Delta_{1}=\lambda^{2}-\lambda-\mu^{2}=\lambda^{2}-(2 \omega)^{-2}, \\
& \Delta_{2}=\lambda^{3}-4 \lambda^{2}-4 \lambda \mu^{2}+4 \lambda+4 \mu^{2}=\lambda^{3}-\omega^{-2} \lambda+\omega^{-2} .
\end{aligned}
$$

Все корни последних уравнений вещественны и различны.

Для функции (8) при условии существования полиномиальных решений имеет место представление

$$
\Phi(z)=z^{-n-1} \frac{E(z)}{E\left(z^{-1}\right)} \cdot \frac{Q_{(a, b)}^{(+)}(z)}{Q_{(a, b)}^{(-)}(z)}
$$

где

$$
\begin{aligned}
Q_{(a, b)}^{( \pm)}(z) & =(\epsilon \mp i) a+2 \omega e^{\mu\left(z+z^{-1}\right)}\left(E(z) E\left(z^{-1}\right)\right)^{-1} b+\left(\epsilon \mathcal{I}_{n}\left(z^{\mp 1}\right) \mp i \mathcal{I}_{n}\left(z^{ \pm 1}\right)\right) b \\
\epsilon^{2} & =1 \\
\mathcal{I}_{n}(z) & =\int_{1}^{z} z^{n} e^{\mu\left(z+z^{-1}\right)} E(z)^{-2} d z .
\end{aligned}
$$

где $a, b$ - вещественные константы. Если выбрать

$$
a=-E(1)^{-2} \sin \frac{\varphi_{0}}{2}, \quad b=\frac{1}{2 \omega} e^{-2 \mu}\left(\cos \frac{\varphi_{0}}{2}+\epsilon \sin \frac{\varphi_{0}}{2}\right),
$$

то $\Phi(1)=e^{i \varphi_{0}}$, т. е. мы получаем решение задачи Коши для уравнения (1) с начальными данными $\varphi(0)=\varphi_{0}$.

Отметим, что, как уже упоминалось, при $l>0$ уравнение $(2)$ не может иметь полиномиальных решений. Это следует, в частности, из уравнений (34), которым удовлетворяют коэффициенты ряда Тейлора для функции $E(z)$. Действительно, если $E(z)$ есть полином, то при некотором $k_{0}>0$ все $a_{k}=0$ при $k \geqslant k_{0}$. Но тогда при $l \geqslant 0$ из (34) будет следовать, что вообще все $a_{k}=0$ при $k \geqslant 0$. Это легко доказывается с помощью математической индукции.

В исключенном в теореме 6 случае, когда для заданных значений параметров уравнение (4) обладает полиномиальным решением, для уравнения (2), по-видимому, 
уже не существуют аналитические в нуле решения. А именно, можно показать, что если

$$
\operatorname{det}\left|I_{k-m-1}(x)\right| \neq 0 \quad \text { при всех } \quad x>0 \quad \text { и } \quad k, m=0,1, \ldots, l-1,
$$

где $I_{\star}(\cdot)$ - модифицированная функция Бесселя первого рода (см. с. 374-377 в [24]), то из существования полиномиального решения уравнения (4) вытекает, что уравнение (2) не имеет решений, аналитических в нуле.

Выполнение условия (53) очевидно при $l=1$, поскольку оно сводится к условию $I_{1}(x) \neq 0$ при $x>0$, что является хорошо известным фактом в теории функций Бесселя. При $l=2$ условие (53) легко выводится из формулы

$$
I_{k}(x) I_{m}(x)=2 \pi^{-1} \int_{0}^{\pi / 2} I_{k+m}(2 x \cos \theta) \cos (k-m) \theta d \theta,
$$

также установленной в этой теории (см. [25], раздел 13.72, формула (2)). При $l>2$ доказательство условия (53) в настоящее время отсутствует, но в пользу его справедливости свидетельствуют результаты численных расчетов.

ГиПотезА 3. Неравенство (53) выполняется при всех неотрицателъных целых $l$.

\section{5. РЕШЕНИЯ, СООТВЕТСТВУЮЩИЕ ОСИ СИММЕТРИИ $B=0$ ОБЛАСТЕЙ ЗАХВАТА ФАЗЫ}

При $B=0$ уравнение (1) преобразуется в уравнение $(2)$ с параметром $l=0$. Наряду со свойствами, присущими всем целым $l$, этот случай обладает также некоторой спецификой, которая связана с тем, что уравнения (2) и (4) в этом случае совпадают. Имеют место следующие результаты.

ТеОрема 7. Пусть $l=0$. Тогда справедливы следующие утвержсдения.

1. Уравнение (2) не имеет решений, представимых в виде $\sum_{k \in \mathbb{Z}} a_{k} z^{k}$, где $a_{k}=$ $a_{k+1}=0$ для некоторого $k$ и $a_{j} \neq 0$ для некоторого $j$, удовлетворяющего условию $j k>0, u$, в частности, когда $k$ пробегает конечное подмножество в $\mathbb{Z}$.

2. Гипотеза 2 верна.

3. Для любого решения $E(z)$ уравнения (2) функции

$$
\begin{gathered}
z^{-1}\left(E^{\prime}\left(z^{-1}\right)-\mu E\left(z^{-1}\right)\right), \quad z e^{\mu\left(z+z^{-1}\right)}\left(E^{\prime}(-z)-\mu E(-z)\right), \\
e^{\mu\left(z+z^{-1}\right)} E\left(-z^{-1}\right),
\end{gathered}
$$

также являются решением этого уравнения.

4. Для голоморфного решения $E(z)$ уравнения (2) любые две функции из четверки

$$
\begin{gathered}
E(z), \quad z^{-1}\left(E^{\prime}\left(z^{-1}\right)-\mu E\left(z^{-1}\right)\right), \\
z e^{\mu\left(z+z^{-1}\right)}\left(E^{\prime}(-z)-\mu E(-z)\right), \quad e^{\mu\left(z+z^{-1}\right)} E\left(-z^{-1}\right)
\end{gathered}
$$

линейно независимы, а любые три - линейно зависимы.

5. Поведение голоморфного решения $E(z)$ уравнения (2) при $z \rightarrow \infty$ определяется его поведением вблизи нуля в соответствии с тождеством

$$
E(z)=2 \omega a z^{-1}\left(E^{\prime}\left(z^{-1}\right)-\mu E\left(z^{-1}\right)\right)+b e^{\mu\left(z+z^{-1}\right)} E\left(-z^{-1}\right),
$$

имеющим место при некоторых ненулевых вещественных $a, b$, зависящих от постоянных параметров и связанных соотношением $a^{2}+b^{2}=1$. 
6. Если аналитическая функиия $E(z)$ удовлетворяет соотношению (56) для некоторых $a \neq 0$ и $b$ таких, что $a^{2}+b^{2}=1$, то $E(z)$ является решением уравнения (2).

7. Если уравнение (2) обладает решением $E(z)$ в виде ряда Лорана с центром в нуле, где-либо сходящимся, то $E(z)=E_{1}(z)+E_{2}(z)$, где $E_{1}(z)$ и $E_{2}(z)-$ суммъ слагаемых с неотрицательными показателями, сходящиеся всюду, и с отрицательными показателями, сходящиеся всюду, кроме нуля, соответственно, которые удовлетворяют этому уравнению порознь. При этом $E_{2}(z)$ с точностью до постоянного множителя совпадает с $z^{-1}\left(E_{1}^{\prime}\left(z^{-1}\right)-\mu E_{1}\left(z^{-1}\right)\right)$. Как следствие, уравнение (2) допускает решение в виде ряда Лорана с центром в нуле тогда и только тогда, когда оно обладает голоморфным решением.

ДокАЗАТЕЛЬСтво. То, что уравнение (2) не имеет полиномиальных решений при $l=0$, следует из уравнений вида (34), рассматриваемых при всех целых $k$, которым, как легко проверить, должны удовлетворять коэффициенты $a_{k}$ разложения $E(z)=\sum_{k \in \mathbb{Z}} a_{k} z^{k}$ любой функции, ему удовлетворяющей. Случай $l=0$ отличается от остальных тем, что для него бесконечная "в обоих направлениях" система (34) распадается, без каких-либо предположений о значениях неизвестных, на две полностью независимые "однонаправленно-бесконечные" системы для подпоследовательностей $a_{k}$ c $k \geqslant 0$ и $k<0$ соответственно. Для этих двух подпоследовательностей имеют место обратные рекуррентные зависимости (вытекающие из (34))

$$
a_{k-1}=(k \mu)^{-1}\left(\lambda+k^{2}\right) a_{k}+k^{-1}(k+1) \mu a_{k+1}, \quad k=1,2, \ldots,
$$

И

$$
a_{k+1}=-((k+1) \mu)^{-1}\left(\lambda+k^{2}\right) a_{k}+(k+1)^{-1} k a_{k-1}, \quad k=-2,-3, \ldots .
$$

Для любой из двух указанных выше подпоследовательностей задание значений каких-либо двух соседних элементов однозначно определяет все предыдущие элементы с неотрицательными индексами (для случая (57)) либо все последующие элементы с отрицательными индексами (для случая (58)). И, очевидно, когда эти значения равны нулю, все остальные указанные элементы также равны нулю. Это и происходит в том случае, когда предполагается, что лишь конечное множество коэффициентов $a_{k}$ могут быть отличны от нуля. Если же хотя бы один из коэффициентов $a_{k}$ нулю не равен, то имеются не равные нулю коэффициенты со сколь угодно большими по величине индексами, положительными, если индекс ненулевого элемента неотрицателен, или отрицательными, если индекс ненулевого элемента отрицателен (и никакие два "соседних" элемента не равны нулю одновременно). Этим утверждение п. 1 доказано.

Утверждение п. 3 можно проверить непосредственным вычислением. Кроме того, оно является проявлением в частном случае значения $l=0$ свойств операторов $\downarrow, b, \diamond$, рассматривавшихся выше. Отличие от общего случая состоит в том, что здесь уравнения (2) и (4) совпадают; соответственно, отображаемое пространство для операторов $b, \diamond$ совпадает со своими образами. Таким образом, отображения $b \equiv d$ и $\diamond$ представляют собой, как и $\downarrow$, эпиморфизмы на пространстве решений уравнения (2). 
Одно из утверждений п. 3 напрямую вытекает из данного факта. А именно, заведомая линейная зависимость любых трех из перечисленных нами функций, которые все удовлетворяют уравнению (2), эквивалентна двумерности линейного пространства решений линейного уравнения второго порядка (2).

Что касается линейной независимости всех пар данных функций, то она доказывается для каждой пары способом, уже применявшимся нами ранее. Докажем, например, линейную независимость функций $z^{-1}\left(E^{\prime}\left(z^{-1}\right)-\mu E\left(z^{-1}\right)\right)$ и $e^{\mu\left(z+z^{-1}\right)} E\left(-z^{-1}\right)$. Заменим сначала для упрощения обозначений $z$ на $z^{-1}$ и предположим, что они все-таки линейно зависимы. Иными словами, предполагается, что линейно зависимы функции $z\left(E^{\prime}(z)-\mu E(z)\right)$ и $e^{\mu\left(z+z^{-1}\right)} E(-z)$. Умножив их на $e^{-\mu z} E(-z)^{-1}$, получаем линейную зависимость функций $z e^{-\mu z} E(-z)^{-1}\left(E^{\prime}(z)-\mu E(z)\right)$ и $e^{\mu z^{-1}}$. Мы знаем, что $E(0) \neq 0$. Поэтому первая функция является аналитической в точке $z=0$ и некоторой ее окрестности. Для второй функции точка $z=0$ сингулярная. Следовательно, линейная зависимость (в данном случае совпадение с точностью до ненулевого постоянного множителя) невозможна. Мы пришли к противоречию.

Рассмотрение остальных пар функций из четверки (55) проводится аналогично.

Формула (56) из п. 5 является прямым следствием п. 4; то же самое касается необращения в нуль констант $a, b$.

Обозначим разность правой и левой частей уравнения (56) как

$$
O_{a, b}(z):=-E(z)+2 \omega a z^{-1}\left(E^{\prime}\left(z^{-1}\right)-\mu E\left(z^{-1}\right)\right)+b e^{\mu\left(z+z^{-1}\right)} E\left(-z^{-1}\right) .
$$

Как только что было отмечено, если $E(z)$ есть голоморфное решение уравнения (2) при $l=0$, то существуют такие ненулевые вещественные $a, b$, что $O_{a, b}(z) \equiv 0$. Теперь, однако, мы будем считать константы $a, b$ (а также параметры $\omega, \lambda, \mu$ ) произвольными вещественными и не будем предполагать, что аналитическая функция $E(z)$ удовлетворяет какому-либо уравнению. В таких условиях имеет место следующее непосредственно проверяемое тождество:

$$
\begin{aligned}
& 2 \omega z a \partial O_{a, b}(z)-2 \omega \mu z a O_{a, b}(z)+b O_{a, b}(-z)+O_{a, b}\left(z^{-1}\right)+(2 \omega a)^{2}\left(\left.\mathfrak{L}\right|_{0} E\right)\left(z^{-1}\right) \equiv \\
& \equiv\left(-1+b^{2}+4 \omega^{2}\left(\lambda+\mu^{2}\right) a^{2}\right) E\left(z^{-1}\right) .
\end{aligned}
$$

Здесь, как и выше, $\partial=d / d z$ и $\left.\mathfrak{L}\right|_{0}:=z^{2} \partial^{2}+\mu\left(1-z^{2}\right) \partial+(-\mu z+\lambda)$ есть оператор левой части уравнения (2), в данном случае взятый при значении $l=0$.

Из данного тождества следует, что при $\left.O_{a, b}(z) \equiv 0 \equiv \mathfrak{L}\right|_{0} E(z)$ для констант выполняется уравнение $-1+b^{2}+4 \omega^{2}\left(\lambda+\mu^{2}\right) a^{2}=0$, и, поскольку $4 \omega^{2}\left(\lambda+\mu^{2}\right)=1$ согласно определению $\lambda$, имеет место равенство $a^{2}+b^{2}=1$, что и утверждается В П. 5 .

Если же мы потребуем, помимо выполнения соотношения (56), т. е. тождества $O_{a, b}(z) \equiv 0$, также выполнения равенства $a^{2}+b^{2}=1$, то из тождества (59) будет следовать $\left.\mathfrak{L}\right|_{0} E(z) \equiv 0$, т. е. выполнение уравнения (2). Это утверждение п. 6 . 
Рассмотрим утверждение п. 7. Если уравнение обладает решением, представимым в виде где-либо сходящегося ряда Лорана с центром в нуле, то коэффициенты $a_{k}$ этого ряда удовлетворяют системе уравнений, состоящей из уравнений (34), взятых при $l=0$ и всех $k \in \mathbb{Z}$. Но, как отмечалось выше, при $l=0$ такая система распадается на две непересекающиеся подсистемы, в одну из которых входят только коэффициенты $a_{k}$ при $k \geqslant 0$, а во вторую - только коэффициенты при $k<0$. Соответственно, если мы модифицируем исходный ряд Лорана, заменив либо все $a_{k}$ при $k \geqslant 0$, либо все $a_{k}$ при $k<0$ нулями, то, во-первых, полученное множество коэффициентов $a_{k}$ будет определять по-прежнему сходящийся степенной ряд и, во-вторых, система уравнений (34) для $l=0, k \in \mathbb{Z}$ по-прежнему будет удовлетворяться. Это означает, что при втором выборе мы получаем решение уравнения (2) (обозначим его $E_{1}(z)$ ), представимое в виде сходящегося где-то ряда Тейлора. Тогда этот ряд будет сходиться при любом $z$, представляя собой голоморфное решение уравнения (2) (это последнее утверждение п. 7). При первом выборе получится решение в виде ряда Тейлора по степеням $z^{-1}$. Совместно с предыдущим оно образует базис двумерного пространства решений уравнения (2). Тем же свойством обладает и пара функций $E_{1}(z)$ и $z^{-1}\left(E_{1}^{\prime}\left(z^{-1}\right)-\mu E_{1}(z)^{-1}\right)$. Поэтому второе решение $E_{2}(z)$ (часть исходного ряда Лорана только с отрицательными степенями) с точностью до числового множителя совпадает с $z^{-1}\left(E_{1}^{\prime}\left(z^{-1}\right)-\mu E_{1}(z)^{-1}\right)$. Оно определено (т. е. ряд сходится) при всех $z$, кроме нуля. Возвращаясь назад, несложно убедиться, что все утверждения п. 7 теперь установлены.

Остается еще п. 2. Бо́льшая часть его утверждений является частным случаем уже установленных закономерностей, справедливых для произвольного $l \geqslant 0$. Но имеется и нетривиальный момент, а именно необходимость выполнения уравнения $\xi(\lambda, \mu)=0$ для существования голоморфного решения (ранее нами была установлена лишь его достаточность). Для ее доказательства нам потребуется следующая лемма.

ЛЕмма 5. Если уравнение при заданных значениях параметров $\mathfrak{L}_{0} E(z)=0$ обладает голоморфным решением, то неоднородное уравнение вида

$$
\mathfrak{L}_{0} G(z)=1
$$

не имеет решений, аналитических в точке $z=0$.

Данная лемма является специальным аналогом леммы 7 , доказываемой в приложении Б, и ее доказательство представляет собой, по существу, упрощенный вариант доказательства леммы 7. Формальные изменения состоят в том, что мы должны, во-первых, подставить в формулах значение $l=0$ и, во-вторых, заменить символ $\widehat{E}$, где он встречается, на $E$. Это вполне естественно, поскольку при $l=0$ уравнения $(2)$ и (4) совпадают. Для обоснования корректности данной замены можно сослаться на п. 3, 4 доказываемой теоремы.

Неформальным в рассматриваемой модификации доказательства леммы 7 является лишь следующее обстоятельство: условие неполиномиальности решения $\widehat{E}(z)$ (теперь это $E(z))$ становится здесь излишним. Во-первых, требовать это специально, как это делается в условиях леммы 7, нет необходимости, поскольку несуществование полиномиальных решений - суть части утверждения п. 1 теоремы. Во-вторых, единственно необходимый в данной ситуации факт необращения в нуль коэффициента $\hat{a}_{l}$, роль которого перешла к $a_{0}$, уже не требует отдельного доказательства, 


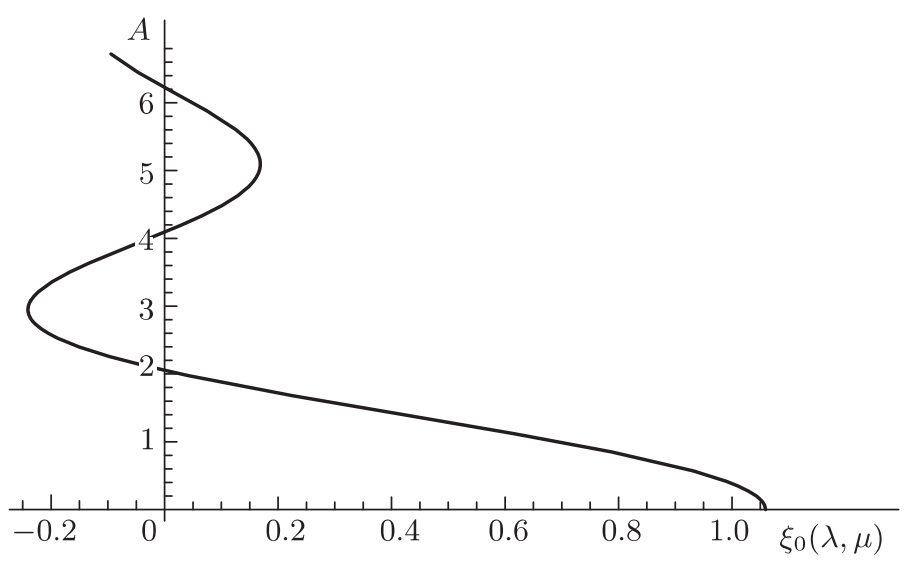

Рис. 2. График значений функции $\xi_{0}(\lambda, \mu)$ (см. (31), значения отложены по горизонтальной координатной оси), в которой аргументы $\lambda, \mu$ выражены через параметр $A$ (см. (3), значения отложены по вертикальной координатной оси), при $\omega=0.7$.

поскольку для $a_{0}$ это есть одно из утверждений теоремы 2. Тем самым справедливость леммы 5 можно считать установленной.

Теперь утверждение о необходимости выполнения уравнения (31) для существования аналитического в нуле решения уравнения (2) вытекает из теоремы 8 (см. приложение Б), а точнее, имеет доказательство в точности такое же, за тем лишь исключением, что оно ссылается в данном случае на лемму 5 вместо леммы 7 .

Теорема доказана.

Рассмотрим простой пример, демонстрирующий применение полученных зависимостей. Ограничиваясь, как и в теореме 7 , случаем $l=0$, рассмотрим поведение функции $\xi_{l}$, вычисляемой непосредственно в соответствии со своим определением (31), на некотором примыкающем к нулю интервале изменения $\mu$. Для “одномерности" задачи зафиксируем значение параметра $\omega=0.7$. Наконец, чтобы получить возможность сопоставления результатов с материалом, отображенным на рис. 1, будем, во-первых, использовать как свободную переменную вместо $\mu$ параметр $A$ и, во-вторых, направим ось $A$ вертикально, а ось значений $\xi_{0}(\lambda, \mu)$ - горизонтально. Требуемый пересчет значений параметров элементарен и производится с помощью формул (3).

Результат вычислений показан на рис. 2. Как оказывается, функция $\xi_{0}$ описывает затухающие осцилляции и, соответственно, обращается в отдельных точках в нуль. Согласно теореме 7 этим корням функции $\xi_{0}$ должны соответствовать голоморфные решения уравнения (2) (в этом можно убедиться, вычислив коэффициенты ряда (32) и подставив их в уравнения (34)), а также в соответствии с изложенным в разделе 1 им соответствуют "перемычки" на цепочке зон захвата фазы, изображенных на рис. 1, расположенные на вертикальной оси координат. Отдельное вычисление дает следующие три первых корня функции $\xi_{0}(\lambda, \mu)$, изображенной на рис. 2 $(\lambda, \mu$ - известные функции $A$, см. (3)): $A=2.05265 \ldots, 4.09182 \ldots, 6.22422 \ldots$. Они полностью соответствуют рис. 1 , полученному на совершенно иной основе. 
ПРИЛОЖЕНИЕ А

\section{Доказательство существования бесконечных произведений (30)}

Если ввести обозначение $R_{m}^{[k]}:=\prod_{j=m}^{k} M_{j}$ для конечных матричных произведений, определяющих матрицы $R_{m}(30)$ как пределы $R_{m}=\lim _{k \rightarrow \infty} R_{m}^{[k]}$, то имеет место рекуррентная зависимость вида $R_{m}^{[k]}=R_{m}^{[k-1]} M_{k}$. Вследствие этого существование бесконечных произведений матриц $R_{m}$ будет вытекать из следующего результата.

ЛЕмма 6. Пусть четыре последовательности вещественных чисел, $\alpha_{j}, \beta_{j}, \gamma_{j}, \delta_{j}$ удовлетворяют рекуррентным соотношениям

$$
\left(\begin{array}{cc}
\alpha_{j} & \beta_{j} \\
\gamma_{j} & \delta_{j}
\end{array}\right)=\left(\begin{array}{cc}
\alpha_{j-1} & \beta_{j-1} \\
\gamma_{j-1} & \delta_{j-1}
\end{array}\right)\left(\begin{array}{cc}
1+\lambda / Y_{j} & \mu^{2} / Y_{j} \\
1 & 0
\end{array}\right),
$$

где $Y_{j}=j(j+l)$. Тогда они сходятся при $j \rightarrow \infty$. При этом $\lim \beta_{j}=0=\lim \delta_{j}$, и для любого целого положительного $j$, большего некоторого $j_{0}$, зависящего от $\lambda, \mu, l, u$ некоторого положительного $N$, также зависящего от $\lambda, \mu, l$, выполняются неравенства

$$
\begin{gathered}
\left|\alpha_{j}-\lim _{j^{\prime} \rightarrow \infty} \alpha_{j^{\prime}}\right|<N \max \left(\left|\alpha_{j_{0}-1}\right|,\left|\alpha_{j_{0}-2}\right|\right) j^{-1} \\
\left|\gamma_{j}-\lim _{j^{\prime} \rightarrow \infty} \gamma_{j^{\prime}}\right|<N \max \left(\left|\gamma_{j_{0}-1}\right|,\left|\gamma_{j_{0}-2}\right|\right) j^{-1}
\end{gathered}
$$

ДокАЗАтЕЛьство ${ }^{5)}$. Прежде всего заметим, что из уравнения (61) следуют равенства

$$
\beta_{j}=\mu^{2} Y_{j}^{-1} \alpha_{j-1}, \quad \delta_{j}=\mu^{2} Y_{j}^{-1} \gamma_{j-1} .
$$

Поскольку $Y_{j}^{-1}=O\left(j^{-2}\right) \rightarrow 0$ при $j \rightarrow \infty$, сходимость последовательностей $\beta_{j}, \delta_{j}$ к нулю будет следовать из существования конечных пределов последовательностей $\alpha_{j}, \gamma_{j}$, доказательство чего становится нашей задачей.

Далее, используя приведенные выше представления $\beta_{j}, \delta_{j}$ через $\alpha_{j-1}, \gamma_{j-1}$, легко получить замкнутые системы уравнений (рекуррентные соотношения), которым удовлетворяют по отдельности элементы последовательностей $\alpha_{j}$ и $\gamma_{j}$. В частности, для $\gamma_{j}$ имеем

$$
\gamma_{j}=\left(1+\lambda Y_{j}^{-1}\right) \gamma_{j-1}+\mu^{2} Y_{j-1}^{-1} \gamma_{j-2} .
$$

Для $\alpha_{j}$ рекуррентные соотношения имеют тот же вид. Как мы увидим, сходимость таких последовательностей не зависит от конкретных значений их "начальных" элементов и определяется только видом самого рекуррентного соотношения. Тем самым, мы можем ограничиться рассмотрением только уравнений (64).

Пусть выбрано и фиксировано некоторое положительное значение $j_{0}$. Очевидно, что как следствие линейных однородных уравнений (64) существует "линейное разложение" любого элемента $\gamma_{j}=\gamma_{j_{0}+k}, k=0,1,2, \ldots$, по двум элементам, предшествующим $\gamma_{j_{0}}$. Иными словами, мы можем записать

$$
\gamma_{j_{0}+k}=\left(1+p_{j_{0}, k}\right) \gamma_{j_{0}-1}+q_{j_{0}, k} \gamma_{j_{0}-2}
$$

5) Данное доказательство является адаптацией доказательства аналогичного, но выполняющегося в несколько иных условиях утверждения, приведенного в [23]. 
где “коэффициенты разложения" $p_{j, k}, q_{j, k}$ зависят лишь от значений идентифицирующих их индексов (и не указанных параметров $l, \mu, \lambda$ ), но не от конкретных значений элементов $\gamma_{j_{0}-1}, \gamma_{j_{0}-2}$ "базиса" разложения. Таким образом, последние можно рассматривать как начальные данные дискретной задачи Коши для уравнений (61). Пара $\gamma_{j_{0}-1}, \gamma_{j_{0}-2}$ определяет значения всех остальных элементов последовательности $\gamma_{j}$. Мы будем считать ее каким-либо образом выбранной и фиксированной (тривиальный случай $\gamma_{j_{0}-1}=0=\gamma_{j_{0}-2}$, естественно, сразу исключается).

Коэффициенты $p_{j, k}, q_{j, k}$, содержащие, по-существу, всю информацию о решениях уравнений (61), удовлетворяют своей собственной системе уравнений. Ее можно получить, если заменить в (65) $j_{0}+k$ на $j_{0}+k+1$ и развернуть выражение справа в соответствии с двумя группированиями $\left(j_{0}+1\right)+k=j_{0}+(k+1)$. Условие совпадения результатов ведет, прежде всего, к редукции вида

$$
q_{j_{0}, k+1}=\mu^{2} Y_{j_{0}-1}^{-1}\left(1+p_{j_{0}+1, k}\right)
$$

которая позволяет заменить все $q$-коэффициенты через выражения, содержащие только $p$-коэффициенты. Затем условие совпадения приводит также к рекуррентному уравнению

$$
p_{j_{0}, k+1}=p_{j_{0}+1, k}+\lambda Y_{j_{0}}^{-1}\left(1+p_{j_{0}+1, k}\right)+\mu^{2} Y_{j_{o}}^{-1}\left(1+p_{j_{0}+2, k-1}\right), \quad k=1,2, \ldots,
$$

которое и становится основным объектом нашего рассмотрения. Оно выглядит достаточно похожим на уравнение (64) и не является в целом ни проще, ни сложнее последнего. Преимущество сведения задачи к анализу именно уравнений (67) состоит в том, что, в отличие от (64), для них “начальные данные" являются в определенном смысле "стандартными" или универсальными и сводятся к равенствам

$$
p_{j_{0},-1}=0, \quad p_{j_{0},-2}=-1 \text {. }
$$

Кроме того, можно считать, что

$$
q_{j_{0},-1}=0, \quad q_{j_{0},-2}=1 .
$$

Действительно, это следует автоматически из равенств (65), если потребовать выполнения последних при произвольных $\gamma_{j_{0}-1}, \gamma_{j_{0}-2}$ для $k=-1$ и $k=-2$. (Заметим, что при $k=-2$ значения (68), (69), будучи подставленными в уравнение (66), порождают тривиальное тождество; при $k=-1$ результатом становится новое соотношение $q_{j_{0}, 0}=\mu^{2} Y_{j_{0}-1}^{-1}$.) Кроме того, теперь в интервал изменения индекса $k$, входящего в уравнение (67), можно добавить значения $k=-1$ и $k=0$. Для них имеем

$$
p_{j_{0}, 1}=p_{j_{0}+1,0}+\lambda Y_{j_{0}}^{-1}\left(1+p_{j_{0}+1,0}\right)+\mu^{2} Y_{j_{o}}^{-1}, \quad p_{j_{0}, 0}=\lambda Y_{j_{0}}^{-1}
$$

соответственно.

Важно то, что исходя из (68) можно последовательно вычислить все значения $p_{j, k}, k=1,2, \ldots$, и при любых $j \geqslant j_{0}$. Действительно, равенства (68) определяют два начальных "слоя" $p_{j,-1}$ и $p_{j,-2}$, где $j$ произвольно. Со своей стороны, уравнение (67) позволяет построить любой элемент $p_{\star, k+1}$ следующего “слоя", если известны два предыдущих $p_{\star, k}$ и $p_{\star, k-1}$. Принцип математической индукции приводит к заключению о вычисляемости последовательно всех “вышележащих слоев" $p_{\star, k+2}, p_{\star, k+3}, \ldots$ 
и о единственности результата. В том числе однозначно определены значения коэффициентов $p_{j_{0}, k}$.

При исследовании уравнений (67) оказывается удобным произвести замену неизвестных с помощью перехода к разностям

$$
\Delta p_{j_{0}, k}=p_{j_{0}, k+1}-p_{j_{0}, k}
$$

которые подчиняются рекурсии вида

$$
\Delta p_{j_{0}, k+1}=\Delta p_{j_{0}+1, k}+\lambda Y_{j_{0}}^{-1} \Delta p_{j_{0}+1, k}+\mu^{2} Y_{j_{0}}^{-1} \Delta p_{j_{0}+2, k-1}
$$

и характеризуются "начальными данными"

$$
\Delta p_{j_{0},-2}=1, \quad \Delta p_{j_{0},-1}=\lambda Y_{j_{0}}^{-1}
$$

вытекающими, в свою очередь, из (68), (70). В соотношении (72) $k=-1,0,1, \ldots$. Как и коэффициенты $p_{j_{0}, k}$, разности $\Delta p_{j_{0}, k}$ однозначно определены приведенными выше уравнениями. Очевидно, они представляют собой рациональные функции параметров $\lambda, \mu$ и целочисленного фиксированного параметра $j_{0}$. В частности, первый шаг рекурсии (72), (73) дает

$$
\Delta p_{j_{0}, 0}=\left(\lambda+\left(\lambda^{2}+\mu^{2} Y_{j_{0}+1}\right) Y_{j_{0}}^{-1}\right) Y_{j_{0}+1}^{-1} .
$$

Существенно, что второй индекс у $\Delta$-разностей, входящих в правую часть (72), всегда меньше второго индекса у разности в левой части (72). Это обстоятельство можно рассматривать как подсказку о перспективности применения математической индукции для исследования данного уравнения. Такой подход можно реализовать следующим образом.

Просуммируем подмножество всех уравнений (72), выделяемых одинаковой суммой первого и второго индексов, в данном случае равной $j_{0}+k+1$. Иными словами, мы рассматриваем $k+2$ экземпляров уравнений (72), в которых произведены замены $j_{0} \rightarrow j_{0}+m, k \rightarrow k-m$, где целочисленная переменная $m$ пробегает интервал $0 \leqslant m \leqslant k+1$. Учитывая взаимное сокращение бо́льшей части слагаемых суммы слева и суммы первых слагаемых справа, в результате которого слева останется единственное слагаемое, а также подставляя явное значение "граничного" вклада $\Delta p_{j_{0}+k+2,-1}$ в соответствии со второй из формул $(73)$, получим

$$
\begin{aligned}
\Delta p_{j_{0}, k+1}= & \lambda Y_{j_{0}+k+2}^{-1}+\lambda \sum_{m=0}^{k+1} Y_{j_{0}+m}^{-1} \Delta p_{j_{0}+1+m, k-m}+ \\
& +\mu^{2} \sum_{m=0}^{k+1} Y_{j_{0}+m}^{-1} \Delta p_{j_{0}+2+m, k-1-m} .
\end{aligned}
$$

Здесь в суммах в правой части, как и выше, второй индекс в сомножителях $\Delta p_{\star, \star}$ строго меньше, чем этот индекс в символе разности слева, равный $k+1$. Поэтому мы можем использовать математическую индукцию применительно к уравнениям (75). Действительно, формулы (73) обеспечивают нас "начальными" значениями $\Delta p_{\star, \star}$, соответствующими минимальным значениям $k=-2$ и $k=-1$, и затем формулы (75) 
позволяют вычислить эти разности последовательно для любого $k \geqslant 0$. Общие формулы на этом пути получить вряд ли возможно, но оценку сверху вида

$$
\left|\Delta p_{j_{0}, k}\right|<\tilde{N}\left|Y_{j_{0}+k+1}\right|^{-1}, \quad k>-2,
$$

согласующуюся с $(73),(74)$, обосновать удается. Здесь $\widetilde{N}$ обозначает некоторое положительное число, не зависящее от $j_{0}$ и $k$. Оно должно быть не меньше $|\lambda|$ для согласованности с (73). Ввиду равенства (74) также должно выполняться соотношение

$$
\left|\lambda+\left(\lambda^{2}+\mu^{2} Y_{j_{0}+1}\right) Y_{j_{0}}^{-1}\right| \leqslant \tilde{N} .
$$

Однако

$$
\lambda+\left(\lambda^{2}+\mu^{2} w_{j_{0}+1} W_{j_{0}+1}\right) W_{j_{0}}^{-1} \equiv \lambda+\mu^{2}+\mu^{2}\left(\frac{1}{j_{0}}+\frac{1}{j_{0}+l}\right)+\frac{\lambda^{2}+\mu^{2}}{j_{0}\left(j_{0}+l\right)}
$$

и, соответственно,

$$
\left|\lambda+\left(\lambda^{2}+\mu^{2} Y_{j_{0}+1}\right) Y_{j_{0}}^{-1}\right|<\left|\lambda+\mu^{2}\right|+|\mu|^{2}(1+1)+\left|\lambda^{2}+\mu^{2}\right| \cdot 1 \leqslant|\lambda|+|\lambda|^{2}+4|\mu|^{2}
$$

при выполнении условия

$$
j_{0}>l+1 \text {. }
$$

Таким образом, при $k=0$ мы получаем из (77) и (78) оценку

$$
\tilde{N} \geqslant|\lambda|+|\lambda|^{2}+4|\mu|^{2} \text {. }
$$

Тем самым нам известны условия, достаточные для того, чтобы оценка (76) была справедлива для $k=-1,0$, и мы можем приступить к рассмотрению шага индукции для произвольного $k$.

Мы предполагаем, что оценка (76) выполняется для всех целых $k$ из интервала $-1 \leqslant k \leqslant k^{\prime}$ для некоторого произвольно фиксированного $k^{\prime}$. Заменив в (75) $k$ на $k^{\prime}$, получим

$$
\begin{aligned}
\left|\Delta p_{j_{0}, k^{\prime}+1}\right| & <|\lambda|\left|Y_{j_{0}+k^{\prime}+2}^{-1}\right|+|\lambda| \tilde{N}\left|Y_{j_{0}+k^{\prime}+2}^{-1}\right| \sum_{m=0}^{k^{\prime}+1}\left|Y_{j_{0}+m}^{-1}\right|+|\mu|^{2} \widetilde{N}\left|Y_{j_{0}+k^{\prime}+2}^{-1}\right| \sum_{m=0}^{k^{\prime}+1}\left|Y_{j_{0}+m}^{-1}\right|= \\
& =|\lambda|\left|Y_{j_{0}+k^{\prime}+2}^{-1}\right|+\tilde{N}\left|Y_{j_{0}+k^{\prime}+2}^{-1}\right|\left[|\lambda| \sum_{m=j_{0}}^{j_{0}+k^{\prime}+1}\left|Y_{m}^{-1}\right|+|\mu|^{2} \sum_{m=j_{0}+1}^{j_{0}+k^{\prime}+2}\left|Y_{m}^{-1}\right|\right]= \\
& =\left|Y_{j_{0}+k^{\prime}+2}^{-1}\right|\left(|\lambda|+\tilde{N}|\lambda| \sum_{m=j_{0}}^{j_{0}+k^{\prime}+1}\left|Y_{m}^{-1}\right|+\tilde{N}|\mu|^{2} \sum_{m=j_{0}+1}^{j_{0}+k^{\prime}+2}\left|Y_{m}^{-1}\right|\right)
\end{aligned}
$$

Следующие неравенства выполняются для всех целых $j_{0}$, удовлетворяющих условию (79), являясь следствием неравенств $\left|z_{1}-z_{2}\right| \geqslant\left|z_{1}\right|-\left|z_{2}\right|$ для всех $z_{1}, z_{2} \in \mathbb{C}$ и $\ln (1+x) \leqslant x$ для любого $x \geqslant 0$ :

$$
\begin{aligned}
\sum_{m=j_{0}}^{j_{0}+k^{\prime}+1}\left|Y_{m}\right|^{-1} & =\sum_{m=j_{0}}^{j_{0}+k^{\prime}+1}\left|\left[m+\frac{l}{2}\right]^{2}-\left(\frac{l}{2}\right)^{2}\right|^{-1} \leqslant \\
& \leqslant \sum_{m=j_{0}}^{j_{0}+k^{\prime}+1}\left(\left[m-\frac{l}{2}\right]^{2}-\left(\frac{l}{2}\right)^{2}\right)^{-1}=
\end{aligned}
$$




$$
\begin{aligned}
& =\sum_{m=j_{0}}^{j_{0}+k^{\prime}+1} \frac{1}{[m-a]^{2}-a^{2}} \int_{m}^{m+1} d m^{\prime}< \\
& <\sum_{m=j_{0}}^{j_{0}+k^{\prime}+1} \int_{m}^{m+1} \frac{d m^{\prime}}{\left[m^{\prime}-1-a\right]^{2}-a^{2}}= \\
& =\int_{j_{0}}^{j_{0}+k^{\prime}+2} \frac{d m^{\prime}}{\left[m^{\prime}-1-a\right]^{2}-a^{2}}<\int_{j_{0}}^{\infty} \frac{d m^{\prime}}{\left[m^{\prime}-1-a\right]^{2}-a^{2}}= \\
& =2^{-1} a^{-1}\left[0-\ln \frac{j_{0}-1-2 a}{j_{0}-1}\right]= \\
& =2^{-1} a^{-1} \ln \left(1+\frac{2 a}{j_{0}-1-2 a}\right) \leqslant \frac{1}{j_{0}-1-2 a}=\frac{1}{j_{0}-1-l},
\end{aligned}
$$

где мы ввели временное обозначение $a=l / 2 \geqslant 0$. В вычислении выше мы должны были потребовать наложения ограничения $a \neq 0$. Но если $a=0$, то мы тем же способом получим

$$
\sum_{m=j_{0}}^{j_{0}+k^{\prime}+1}\left|Y_{m}\right|^{-1}<\int_{j_{0}}^{\infty} \frac{d m^{\prime}}{\left[m^{\prime}-1\right]^{2}}
$$

т. е. тот же самый итоговый результат. Тем самым мы получили оценку сверху для значений первой суммы в последней строке в (81). Оценка для второй суммы получается, очевидно, из (82) формальной заменой $j_{0}$ на $j_{0}+1$.

Таким образом, мы получаем, что при условии $j_{0}>1+l$ из неравенства (81) вытекает неравенство

$$
\left|\Delta p_{j_{0}, k^{\prime}+1}\right|<\left|Y_{j_{0}+k^{\prime}+2}^{-1}\right| \frac{|\lambda|+\tilde{N}\left(|\lambda|+|\mu|^{2}\right)}{j_{0}-1-l} .
$$

Несколько усилим теперь ограничение на допустимые значения для $j_{0}$ по сравнению с (79), потребовав выполнения неравенства

$$
j_{0}>2+l+|\lambda|+|\mu|^{2} .
$$

При таком ограничении дробь в правой части (83) не будет превосходить $\tilde{N}$ тогда и только тогда, когда

$$
\tilde{N} \geqslant \frac{|\lambda|}{\left(j_{0}-1-l-|\lambda|-|\mu|^{2}\right)^{-1}} .
$$

С другой стороны, ввиду введенного ограничения на $j_{0}$ (83) знаменатель в (85) всегда больше единицы, и потому (85) заведомо выполнено при $\widetilde{N} \geqslant|\lambda|$. Тем более оно будет выполнено при соблюдении ограничения (80), что предполагалось нами с начала доказательства. Таким образом, при соблюдении (84), (80) мы получаем неравенство

$$
\left|\Delta p_{j_{0}, k^{\prime}+1}\right|<\tilde{N}\left|Y_{j_{0}+k^{\prime}+2}^{-1}\right|
$$

что завершает доказательство возможности осуществления шага индукции при произвольном $k^{\prime}$. Справедливость оценок (76), таким образом, доказана. 
Заметим следом, что в качестве $j_{0}$ можно использовать любое число, удовлетворяющее условию (84), без необходимости изменять значение множителя $\widetilde{N}$. Минимальные (пороговые) значения для $j_{0}$ и $\widetilde{N}$ могут зависеть только от параметров $l, \mu, \lambda$.

Используем теперь (76) для доказательства сходимости рассматриваемой последовательности.

Из (76) и (82) следует, что ряд $\sum_{k=0}^{\infty} \Delta p_{j_{0}, k}$ обладает сходящейся мажорантой $\widetilde{N} \sum_{k}^{\infty}\left|Y_{j_{0}+k+1}\right|^{-1}$ и, таким образом, абсолютно сходится. Это означает, что последовательность его частных сумм $\sum_{m=0}^{l} \Delta p_{j_{0}, k}=p_{j_{0}, k+1}-p_{j_{0},-1}=p_{j_{0}, k+1}$ также сходится при $k \rightarrow \infty$. Более того, из (76) и (82) следует, что

$$
\left|p_{j_{0}, k+1}\right| \leqslant \widetilde{N} \sum_{m=0}^{k}\left|Y_{j_{0}+m+1}\right|^{-1}=\tilde{N} \sum_{m=j_{0}+1}^{k+j_{0}+1}\left|Y_{m}\right|^{-1}<\tilde{N}\left(j_{0}-l\right)^{-1}
$$

равномерно по $k$. Отсюда, в частности, имеем

$$
\left|\lim _{k^{\prime} \rightarrow \infty} p_{j_{0}, k^{\prime}}\right| \leqslant \widetilde{N}\left(j_{0}-l\right)^{-1}
$$

причем “скорость” сходимости можно охарактеризовать следующим образом:

$$
\begin{aligned}
\left|\lim _{k^{\prime} \rightarrow \infty} p_{j_{0}, k^{\prime}}-p_{j_{0}, k}\right| & =\left|\sum_{m=k}^{\infty} \Delta p_{j_{0}, m}\right| \leqslant \tilde{N} \sum_{m=k}^{\infty}\left|Y_{j_{0}+m}^{-1}\right|= \\
& =\tilde{N} \sum_{m=j_{0}+k}^{\infty}\left|Y_{m}^{-1}\right| \leqslant \tilde{N}\left(j_{0}+k-1-l\right)^{-1} .
\end{aligned}
$$

Далее, в соответствии с (65) и (66)

$$
\gamma_{j}=\gamma_{j_{0}+k}=\left(1+p_{j_{0}, k}\right) \gamma_{j_{0}-1}+\mu^{2} Y_{j_{0}-1}^{-1}\left(1+p_{j_{0}+1, k-1}\right) \gamma_{j_{0}-2}
$$

и сходимость последовательности $\gamma_{j}$ при $j \rightarrow \infty$ следует из сходимости при $k \rightarrow \infty$ выражения в правой части данной формулы, имеющей место как следствие установленной выше сходимости последовательностей $p_{j_{0}, k}$ и, эквивалентно, $p_{j_{0}+1, k}$. Кроме того,

$$
\lim _{j^{\prime} \rightarrow \infty} \gamma_{j^{\prime}}-\gamma_{j}=\gamma_{j_{0}-1}\left(\lim _{k^{\prime} \rightarrow \infty} p_{j_{0}, k^{\prime}}-p_{j_{0}, k}\right)+\mu^{2} Y_{j_{0}-1}^{-1} \gamma_{j_{0}-2}\left(\lim _{k^{\prime} \rightarrow \infty} p_{j_{0}+1, k^{\prime}}-p_{j_{0}+1, k}\right),
$$

и, следовательно,

$$
\begin{aligned}
\left|\lim _{j^{\prime} \rightarrow \infty} \gamma_{j^{\prime}}-\gamma_{j}\right| \leqslant\left|\gamma_{j_{0}-1}\right| \cdot\left|\lim _{k^{\prime} \rightarrow \infty} p_{j_{0}, k^{\prime}}-p_{j_{0}, k}\right|+ & \\
& +\left|\mu^{2} Y_{j_{0}-1}^{-1}\right| \cdot\left|\gamma_{j_{0}-2}\right| \cdot\left|\lim _{k^{\prime} \rightarrow \infty} p_{j_{0}+1, k^{\prime}}-p_{j_{0}+1, k}\right| \leqslant \\
\leqslant & \left(1+\left|\mu^{2} Y_{j_{0}-1}^{-1}\right|\right) \cdot \max _{j^{\prime}=j_{0}-1, j_{0}-2}\left|\gamma_{j^{\prime}}\right| \cdot\left|\lim _{k^{\prime} \rightarrow \infty} p_{j_{0}, k^{\prime}}-p_{j_{0}, k}\right| \leqslant \\
\leqslant & \left(1+\left|\mu^{2} Y_{j_{0}-1}^{-1}\right|\right) \cdot \max _{j^{\prime}=j_{0}-1, j_{0}-2}\left|\gamma_{j^{\prime}}\right| \cdot \widetilde{N}\left(j_{0}+k-1-l\right)^{-1} \leqslant \\
\leqslant & \tilde{N}\left(1+\frac{|\mu|^{2}}{\left(j_{0}-1\right)\left(j_{0}-1-l\right)}\right) \frac{j}{j-1-l} \cdot \max _{j^{\prime}=j_{0}-1, j_{0}-2}\left|\gamma_{j^{\prime}}\right| \cdot j^{-1}<
\end{aligned}
$$




$$
\begin{aligned}
& <\tilde{N}\left(1+\frac{|\mu|^{2}}{\left(j_{0}-1\right)\left(j_{0}-1-l\right)}\right)\left(1+\frac{1+l}{j_{0}-1-l}\right) \cdot \max _{j^{\prime}=j_{0}-1, j_{0}-2}\left|\gamma_{j^{\prime}}\right| \cdot j^{-1}< \\
& <\tilde{N} \cdot 2(2+l) \cdot \max _{j^{\prime}=j_{0}-1, j_{0}-2}\left|\gamma_{j^{\prime}}\right| \cdot j^{-1}
\end{aligned}
$$

где учтено ограничение (65), из которого следует, что

$$
\left(j_{0}-1\right)\left(j_{0}-1-l\right)>\left(1+l+|\lambda|+|\mu|^{2}\right)\left(1+|\lambda|+|\mu|^{2}\right)>|\mu|^{2},
$$

и то, что $j \geqslant j_{0}$. Это второе из неравенств (62) для

$$
N=\tilde{N} \cdot 2(2+l)>2(2+l)\left(|\lambda|+|\lambda|^{2}+4|\mu|^{2}\right) .
$$

Как отмечалось выше, для последовательности $\alpha_{j}$ имеем те же уравнения, что и для $\gamma_{j}$, и такое же рассуждение приводит к аналогичной оценке - первому из неравенств (62). Лемма доказана.

Применяя лемму 6 для обоснования свойства сходимости последовательности матриц $R_{m}^{[k]}$, мы фиксируем числа $\alpha_{m}, \beta_{m}, \gamma_{m}, \delta_{m}$ равенством

$$
\left(\begin{array}{ll}
\alpha_{m} & \beta_{m} \\
\gamma_{m} & \delta_{m}
\end{array}\right)=R_{m}^{m}=M_{m}
$$

и затем считаем, что все $\alpha_{j}, \beta_{j}, \gamma_{j}, \delta_{j}$ при $j>m$ вычисляются с помощью формулы (61). Тогда в соответствии с леммой пределы этих последовательностей существуют (их можно обозначить как $\alpha_{\infty}, \gamma_{\infty}$, предел последовательностей $\beta_{j}, \delta_{j}$ есть ноль), и они определяют элементы матрицы $R_{m}$. Далее, если ограничиться рассмотрением индексов $m$, не меньших, чем числа в правой части неравенства (84), то будет иметь место неравенство $\left\|R_{m}^{[j]}-R_{m}\right\|<N^{\prime} j^{-1}$ для некоторого $N^{\prime}$, зависящего только от параметров $l, \lambda, \mu$, где под нормой $\|\ldots\|(2 \times 2)$-матрицы понимается максимум модулей элементов ее первого столбца. В частности, неравенство имеет место и при $j=m$. Таким образом, при достаточно большом $m$ элементы матриц $R_{m}^{[m]}$ и $R_{m}$ сколь угодно близки. С другой стороны, при достаточно большом $m$ сколь угодно близки элементы матриц $R_{m}^{[m]}=M_{m}$ и $\lim _{m \rightarrow \infty} M_{m}=: M_{\infty}:=\left(\begin{array}{ll}1 & 0 \\ 1 & 0\end{array}\right)$. Следовательно, сколь угодно близкими будут и матрицы $R_{m}$ и $M_{\infty}$, так что имеем, по существу, как следствие леммы 6 следующее утверждение.

СлеДСТвИЕ 2. Имеет место равенство $\lim _{m \rightarrow \infty} R_{m}=M_{\infty}$; соответственно, модули всех элементов всех матрии, $R_{m}$ ограничены общим числом.

\section{ПРИЛОЖЕНИЕ Б}

\section{Гипотеза 2 есть следствие гипотезы 1}

ЛЕмма 7. Пусть $l \in \mathbb{N}$. Если однородное уравнение (4) обладает решением, аналитическим в нуле, не являющимся полиномом от переменной $z$, то неоднородное уравнение

$$
\mathfrak{L} G(z) \equiv z^{2} G^{\prime \prime}(z)+\left((l+1) z+\mu\left(1-z^{2}\right)\right) G^{\prime}(z)+(-\mu(l+1) z+\lambda) G(z)=1
$$

не имеет решений, аналитических в нуле. 
ДокАЗАтЕльство. Если известны два линейно независимых решения $E_{1}(z), E_{2}(z)$ однородного уравнения $\mathfrak{L} E(z)=0$ (т. е. уравнения $(2)$ ), то одно из решений уравнения (92) определяется квадратурой (неопределенным интегралом) вида

$$
G(z)=\int^{z} \frac{E_{1}(z) E_{2}(\zeta)-E_{1}(\zeta) E_{2}(z)}{E_{1}^{\prime}(\zeta) E_{2}(\zeta)-E_{1}(\zeta) E_{2}^{\prime}(\zeta)} \frac{d \zeta}{\zeta^{2}}
$$

Вронскиан $E_{1}^{\prime}(\zeta) E_{2}(\zeta)-E_{1}(\zeta) E_{2}^{\prime}(\zeta)$, являющийся знаменателем подынтегрального выражения, в нашем случае есть известная функция. Она определяется формулой вида (37), где $W_{+}(z):=W_{+}$в данном случае есть некоторая ненулевая константа, значение которой зависит от конкретного выбора функций $E_{1}, E_{2}$ (оно нам здесь не понадобится). Все остальные решения уравнения (92) получаются из указанного с помощью добавления произвольной линейной комбинации функций $E_{1}(z)$ и $E_{2}(z)$ с постоянными коэффициентами, что эквивалентно различным выборам двух произвольных констант интегрирования (на самом деле (93) есть разность двух интегралов).

Мы можем выбрать

$$
E_{1}(z)=2 \omega z^{-l+1} e^{\mu\left(z+z^{-1}\right)}\left(\widehat{E}^{\prime}(-z)-\mu \widehat{E}(-z)\right), \quad E_{2}(z)=e^{\mu\left(z+z^{-1}\right)} \widehat{E}\left(-z^{-1}\right),
$$

где $\widehat{E}(z)$ - аналитическое в нуле решение уравнения (4) (см. лемму 3). После подстановки данных представлений в (93) получим

$$
W_{+}^{-1} G(z)=e^{\mu\left(z+z^{-1}\right)}\left(z^{-l+1}\left(\widehat{E}^{\prime}(-z)-\mu \widehat{E}(-z)\right) J_{1}(z)-\widehat{E}\left(-z^{-1}\right) J_{2}(z)\right)
$$

где

$$
J_{1}(z)=\int^{z} \zeta^{l-1} \widehat{E}\left(-\zeta^{-1}\right) d \zeta, \quad J_{2}(z)=\int^{z}\left(\widehat{E}^{\prime}(-\zeta)-\mu \widehat{E}(-\zeta)\right) d \zeta
$$

Функция $J_{2}(z)$, очевидно, аналитическая в точке $z=0$. Рассмотрим функцию $J_{1}$. Здесь подынтегральное выражение может быть разложено в ряд Лорана следующим образом:

$$
\zeta^{l-1} \widehat{E}\left(-\zeta^{-1}\right)=\sum_{k=0}^{\infty}(-1)^{k} \hat{a}_{k} \zeta^{l-1-k}=\sum_{k=1-l}^{\infty}(-1)^{k+l+1} \hat{a}_{k+l-1} \zeta^{-k}
$$

Для $l>1$ коэффициент при $z^{-1}$ (вычет) в разложении $\zeta^{l-1} \widehat{E}\left(-\zeta^{-1}\right)$ равен

$$
(-1)^{l} \hat{a}_{l}=(-1)^{l}(l !)^{-1} \partial^{l} \widehat{E}(0)=(-1)^{l}(l !)^{-1} E(0) .
$$

Он не равен нулю. Действительно, если $E(0)=a_{0}=0$, тогда вследствие первого из уравнений (34) справедливо также и $a_{1}=0$. Соответственно, остальная последовательность уравнений (34) дает нулевые значения в итоге для всех $a_{k}$. Но это невозможно, так как $E(z)=\partial^{l} \widehat{E}(z) \not \equiv 0$, поскольку $\widehat{E}(z)$ не является полиномом. Следовательно, $\hat{a}_{l} \neq 0$.

Если же $l=1$, то вычет равен $-\hat{a}_{1}=-\widehat{E}^{\prime}(0)$. Вообще говоря, эта производная может обращаться в нуль, но тогда вследствие соотношения $\mu \widehat{E}^{\prime}(0)+\lambda \widehat{E}(0)=0$ (результат подстановки $z=0$ в (4)) мы бы имели $\lambda \widehat{E}(0)=0$, и $\lambda=0$, поскольку 
$\widehat{E}(0) \neq 0$. Однако при $l=1$ и $\lambda=0$ уравнение для $\widehat{E}(z)$, приобретающее в таком случае вид $z^{2} \widehat{E}^{\prime \prime}+\mu\left(1-z^{2}\right) \widehat{E}^{\prime}=0$, обладает решением $E=$ const, т. е. полиномиальным решением степени $l-1=0$. Следовательно, исключительный случай $l=1, \lambda=0$ выходит за рамки условий леммы. Тогда мы можем заключить, что при соблюдении предполагаемых условий вычет подынтегрального выражения в интеграле $J_{1}$ всегда не равен нулю. А это означает, что после интегрирования $J_{1}(z)$ будет представлено как сумма сходящегося ряда Лорана и функции $\ln z$ с некоторым ненулевым коэффициентом. Эта функция не является однозначной, и она единственная обладает этим свойством среди всех составляющих элементов выражения (94). В результате $G(z)$ также оказывается многозначной функцией, изменяющей свое значение при обходе нуля по окружности сколь угодно малого радиуса. Следовательно, эта функция не является аналитической в нуле. Все иные решения уравнения (94) получаются прибавлением к $G(z)$ некоторой однозначной функции и также будут обладать этим свойством. Лемма доказана.

Теорема 8. Если при $l \in \mathbb{N}$ и некоторых заданных $\lambda, \mu$ уравнение (4) не допускает полиномиальных решений, то выполнение уравнения (31) является необходимым (и, как было ранее показано, достаточным) условием существования всюду голоморфного решения уравнения (2).

ДокАЗАТЕЛЬство. Если бы теорема не выполнялась, это означало бы, что при заданных $\lambda, \mu$ уравнение (2) обладает голоморфным решением $E(z)$, в то время как уравнение (31) не выполняется. Несмотря на последнее обстоятельство, мы имеем возможность, как и прежде, вычислить коэффициенты $a_{k}$ по формулам (33), причем они будут определять посредством степенного ряда вида (32) аналитическую функцию, которую мы на сей раз обозначим $g(z)$, резервируя символ $E$ за решениями уравнения (2). Согласно способу своего построения функция $g(z)$ не является решением уравнения (92). А именно, ее образом при действии оператора $\mathfrak{L}$ будет не ноль, а ненулевая по предположению величина $\xi_{l}$, представляющая собой константу, если говорить о зависимости от переменной $z$ (см. уравнения (31), (35)). Таким образом, аналитическая в нуле функция $G(z)=\xi_{l}^{-1} g(z)$ оказывается решением уравнения (92). Однако, как мы показали выше, при предполагаемых условиях это уравнение не может иметь аналитических в нуле решений (лемма 7). Полученное противоречие свидетельствует о ложности предположения о невыполнении уравнения (31) для рассматриваемых значений параметров. Теорема доказана.

Представляется уместным отметить здесь следующий факт, легко доказываемый на основании изложенного выше и представляющий определенный самостоятельный интерес.

ЛЕмма 8. Пусть при $l \in \mathbb{N}$ существует аналитическое в нуле решение $\widehat{E}(z) \not \equiv 0$ уравнения (4). Оно является полиномиальным тогда и только тогда, когда $\partial^{l} \widehat{E}(0)=0$.

Действительно, в процессе доказательства леммы 7 было показано, что для неполиномиального аналитического в нуле решения $\widehat{E}(z)$ уравнения (4) коэффициент его разложения в ряд Тейлора $\hat{a}_{l}=(l !)^{-1} \partial^{l} \widehat{E}(0) \neq 0$. С другой стороны, в работе [21] доказано, что полином, удовлетворяющий уравнению (4), имеет степень, в обозначениях настоящей работы равную $l-1$. Следовательно, его производная порядка $l$ есть 
тождественный ноль. Этих соотношений достаточно для выполнения утверждения леммы.

Благодарности. Авторы выражают благодарность А. А. Глуцуку за полезные замечания и предложения, высказанные в ходе обсуждения данной работы. Работа поддержана частично РФФИ (грант № 14-01-00506).

\section{Список литературы}

[1] В. М. Бухштабер, С. И. Тертычный, УМН, 69:2(416) (2014), 201-202.

[2] R. L. Foote, Rep. Math. Phys., 42:1-2 (1998), 249-271, arXiv: math/9808070.

[3] R. L. Foote, M. Levi, S. Tabachnikov, Amer. Math. Monthly, 120:3 (2013), 199-216, arXiv: 1207.0834.

[4] B. D. Josephson, Phys. Lett., 1:7 (1962), 251-253.

[5] W. C. Stewart, Appl. Phys. Lett., 12:8 (1968), 277-280.

[6] D. E. McCumber, J. Appl. Phys., 39:7 (1968), 3113-3118.

[7] А. Бароне, Дж. Патерно, Эффект Джозефсона: физика и применение, Мир, М., 1984.

[8] В. В. Шмидт, Введение в физику сверхпроводников, МСНМО, М., 2000.

[9] J. Guckenheimer, Yu. S. Ilyashenko, Moscow Math. J., 1:1 (2001), 27-47.

[10] D. Schmidt, G. Wolf, "Double confluent Heun equation", Heun's Diffrential Equations, ed. A. Ronveaux, Oxford Univ. Press, Oxford, 1995, 129-188.

[11] С. Славянов, В. Лай, Специальные функиии. Единая теория, основанная на анализе особенностей, "Невский диалект", СПб., 2002.

[12] В. М. Бухштабер, О. В. Карпов, С. И. Тертычный, УМH, 67:1(403) (2012), 181-182.

[13] В. М. Бухштабер, О. В. Карпов, С. И. Тертычный, ТМФ, 162:2 (2010), 254-265.

[14] A. Klimenko, O. Romaskevich, Moscow Math. J., 14:2 (2014), 367-384.

[15] V. M. Buchstaber, O. V. Karpov, S.I. Tertychniy, "Quantum Josephson $D / A$ converter driven by trains of short $2 \pi$-pulses", Abstracts of the II Conference on Precision Electromagnetic Measurements CPEM-2002 (Ottawa, Canada, June 16-21, 2002), ed. U. Feller, IEEE, New York, 2002, 502-503.

[16] В. М. Бухштабер, О. В. Карпов, С. И. Тертычный, УМН, 59:2(356) (2004), 187-188.

[17] А. А. Глуцюк, В.А. Клепцын, Д. А. Филимонов, И. В. Щуров, Функи. анализ и его прил., 48:4 (2014), 47-64.

[18] Ю. С. Ильяшенко, Д.А. Рыжов, Д.А. Филимонов, Функи. анализ и его прил., 45:3 (2011), 41-54.

[19] Д. А. Рыжов, Резонансный захват и специальные эргодические теоремы, Дис. ... канд. физ.-матем. наук, МГУ, М., 2012.

[20] S.I. Tertychniy, The modelling of a Josephson junction and Heun polynomials, arXiv: math-ph/0601064.

[21] В. М. Бухштабер, С. И. Тертычный, ТМФ, 176:2 (2013), 163-188.

[22] S. I. Tertychniy, Long-term behavior of solutions of the equation $\dot{\phi}+\sin \phi=f$ with periodic $f$ and the modeling of dynamics of overdamped Josephson junctions, arXiv: math-ph/0512058.

[23] S. I. Tertychniy, Electron. J. Differential Equations, 2007:133 (2007), 1-20.

[24] M. Abramowitz, I. A. Stegun (eds.), "Modified Bessel Functions I", Handbook of Mathematical Functions with Formulas, Graphs, and Mathematical Tables, Dover, New York, 1972.

[25] Дж.Н. Ватсон, Теория бесселевых функиий, т. 1, ИЛ, М., 1949. 